\title{
Neuroinflammation, Neuroautoimmunity, and the Co-Morbidities of Complex Regional Pain Syndrome
}

\author{
Mark S. Cooper • Vincent P. Clark
}

Received: 17 June 2012 / Accepted: 23 July 2012 / Published online: 25 August 2012

(C) The Author(s) 2012. This article is published with open access at Springerlink.com

\begin{abstract}
Complex Regional Pain Syndrome (CRPS) is associated with non-dermatomal patterns of pain, unusual movement disorders, and somatovisceral dysfunctions. These symptoms are viewed by some neurologists and psychiatrists as being psychogenic in origin. Recent evidence, however, suggests that an autoimmune attack on selfantigens found in the peripheral and central nervous system may underlie a number of CRPS symptoms. From both animal and human studies, evidence is accumulating that neuroinflammation can spread, either anterograde or retrograde, via axonal projections in the CNS, thereby establishing neuroinflammatory tracks and secondary neuroinflammatory foci within the neuraxis. These findings suggest that neuroinflammatory lesions, as well as their associated functional consequences, should be evaluated during the differential diagnosis of non-dermatomal pain presentations, atypical movement disorders, as well as other "medically unexplained symptoms", which are often attributed to psychogenic illness.
\end{abstract}

\footnotetext{
M. S. Cooper $(\triangle)$

Department of Biology, University of Washington,

Seattle, WA 98195-1800, USA

e-mail: mscooper@u.washington.edu

V. P. Clark

Departments of Psychology and Neurosciences,

University of New Mexico,

Albuquerque, NM 87131-0001, USA

V. P. Clark

Mind Research Network

and Lovelace Biomedical Research Institute,

Albuquerque, NM 87106, USA

Keywords Neuropathic pain · Psychogenic · Physiologic . Hysteria $\cdot$ Somatoform $\cdot$ Conversion disorder - Microglia . GABAergic $\cdot$ Glycinergic $\cdot$ Neuroimaging $\cdot$ Reflex sympathetic dystrophy $\cdot$ Charcot $\cdot$ Autoimmune . Neuroautoantibodies $\cdot$ Neuroautoimmunity

Hysteria has its laws, its determination, precisely like a nervous system ailment with a material lesion. Its anatomical lesion still eludes our means of investigation..." Dr. Jean-Martin Charcot, 1890.

\section{Introduction}

Complex Regional Pain Syndrome (CRPS), formerly referred to as Reflex Sympathetic Dystrophy (RSD), is one of the diseases classically defined as hysteria minor by the early neurologist, Dr. Jean-Martin Charcot (1892). To this day, the sensory disorders and movement disorders of CRPS are sometimes diagnosed as somatization disorders, or conversion disorders, respectively (Ochoa and Verdugo 1995; Verdugo and Ochoa 2000; Hawley and Weiner 2011). Such somatoform disorders are defined as a chronic condition where physical symptoms are observed, but no physical cause can be found (Stone et al. 2011). In the absence of medical explanations for the symptoms, a psychological etiology is presumed (Stone et al. 2009, Stone et al. 2010).

In contrast to these views, substantial evidence has been obtained that CRPS is a neuroinflammatory disorder, with a probable autoimmune component in many individuals (Blaes et al. 2007, Goebel et al. 2011; Kohr et al. 2011; Goebel 2011). In a study of adult CRPS patients, $90 \%$ of the cohort had autoantibodies to either the beta(2)-adrenergic receptor ( $\beta 2 \mathrm{AR})$ or the muscarinic acetylcholine receptor (M2R) (Kohr et al. 2011). 
$55 \%$ of the patients had autoantibodies to both neurotransmitter receptors. Integrating these new research findings into neurological and psychiatric practice will require a comparison between historical views on hysteria, contemporary views on psychogenic illness, as well as emerging information about neuroautoimmunity and neuroinflammation.

In the 1880s, Charcot first hypothesized that hysteria was generated by non-structural lesions in the nervous system (Harris 2005). He postulated that these lesions were likely to be biochemical or physiological in character. In describing a case study, during a lecture on hysteria, Charcot (1885) stated: "We have here unquestionably one of those lesions which escape our present means of anatomical investigation, and which, for want of a better term, we designate dynamic or functional lesions."

Charcot's concepts of dynamic and functional lesions are especially useful to utilize when analyzing the local and nonlocal effects of neuroinflammation within the nervous system. Neuroinflammation is now implicated in many neurological and neuropsychiatric disorders that were once classified as hysteria, e.g. dystonia (Sigel et al. 2007; Lee et al. 2009; Simonyan et al. 2010), CRPS (Del Valle et al. 2009), temporal lobe epilepsy (Huberfeld et al. 2007). In this paper, we discuss how neuroinflammatory lesions, together with their functional consequences, are likely to be a subset of the functional and dynamic lesions that were originally postulated by Charcot to explain the etiology of hysteria (1885). In addition, we discuss the need to improve neuroimaging of neuroinflammatory lesions, to help facilitate the diagnosis and mechanistic understanding of neuroinflammation-mediated neurological and neuropsychiatric disorders.

\section{Physiological versus psychogenic symptoms}

In a recent editorial on a paper dealing with medically unexplained movement disorders, Stone and Edwards (2011) make the following comment:

Proving that a symptom affecting a heterogeneous group of people is "psychogenic" using epidemiological data may be as unachievable as trying to prove that it is not. The word psychogenic itself suggests an exclusion of biological factors that is at odds with a biopsychosocial model now prevalent in understanding most mental health disorders and physical symptom syndromes. The use of this term (i.e. psychogenic) may play a role in restricting research scope, and also may adversely affect clinical interest, care, and understanding of these patients. Studies such as this are helpful in challenging stereotypes, and may push us away from a narrow dogmatic approach and toward a broader view of the etiologies and mechanisms of these common and disabling disorders.

In this context, we discuss a number of questions related to CRPS and neuroinflammation. Specifically, does neuroinflammation underlie many of the sensory, autonomic, and movement disorders of CRPS? If so, why have these dysfunctions so often been interpreted as being psychogenic? What diagnostic and mechanistic criteria are being used to make these conclusions? Are the diagnostic criteria for psychogenic illness compatible with emerging views of neuroinflammation and neuroautoimmunity? In the following sections, we discuss how autoimmunity and neuroinflammation may combine to produce some of the most controversial symptoms in neurology and psychiatry: the co-morbidities of CRPS.

Neuroautoimmunity and the initiation of CRPS

Recent discoveries have helped to elucidate possible mechanisms for the initiation and progression of CRPS. In general, neuroautoimmune responses are determined by how infiltrating leukocytes react to autoantibodies, which bind to autoantigens located on the surfaces of neuronal and glial cell targets (Fig. 1). For a substantial fraction of adult CRPS cases (Kohr et al. 2011), initiation of CRPS may lie in a breakdown of immunologic self-tolerance, and the development of autoantibodies to the $\beta 2 \mathrm{AR}$ and M2R neurotransmitter receptors. Once autoantibodies have been generated to these neuroantigens in a given individual, an ongoing progression of stereotyped autoimmune-mediated neuroinflammatory responses might become initiated.
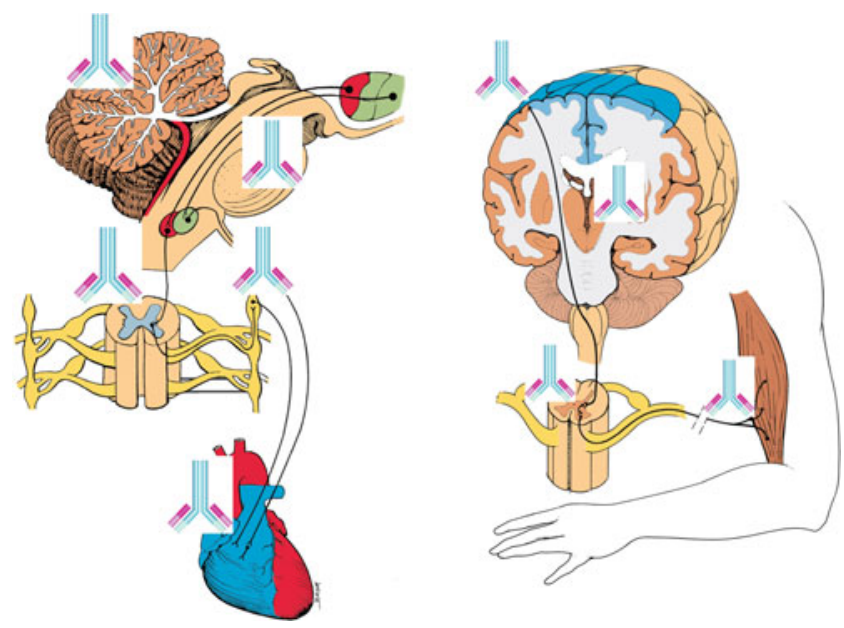

Fig. 1 Potential autoantibody binding sites in CRPS patients for $\beta 2 A R$ (beta(2)-adrenergic receptor) and M2R (muscarinic 2 acetylcholine receptor) autoautoantibodies. (images modified from The Inner Man ${ }^{\mathrm{TM}}$, Medical Illustrations Company LLC). a. $\beta 2 \mathrm{AR}$ and M2R sites in the central nervous system and heart: $\beta 2 \mathrm{AR}$ in cerebellum - (Reznikoff et al. 1986); $\beta 2 A R$ in reticular formation-(Culmsee 2009); $\beta 2$ AR in sympathetic autonomic nervous system - (Katzung 2001), M2R in parasympathetic autonomic system and heart-(Katzung 2001) B2AR also in astrocytes and microglia (Mantyh et al. 1995; Wang et al. 2010) b. M2R distribution in pyramidal motor pathway to skeletal muscles M2R in motor cortex and thalamus - (Levey et al. 1991) M2R in peripheral nerves - (Katzung 2001) 
Achieving a cellular and molecular understanding of the clinical progression of CRPS, as well as the generation of its complex symptoms, requires modeling of the immunologic and integrative physiology involved. It is important to consider how distressed neurons and glial cells release factors that stimulate the extravasation of leukocytes and autoantibodies, from the bloodstream, into the parenchyma of the CNS (Watkins et al. 2007). Serious neuroinflammatory consequences would be expected to arise when $\beta 2 A R$ and M2R autoantibodies exudate from blood vessels, together with complement proteins and leukocytes (Figs. 1 and 2).

Beggs et al. (2010) have recently found that the blood-brain barrier in the spinal cord of rats is transiently compromised in response to peripheral nerve injury. Extravasation of leukocytes into the parenchyma of the cord has been found to last for several days following a sciatic nerve injury (Milligan ED, personal communication). In a CRPS patient with a peripheral nerve injury, one might expect circulating autoantibodies to exudate into the parenchyma of the injured nerve. Autoimmune attack on peripheral nerves might trigger leukocyte extravasation, autoantibody exudation, and neuroimmune activation in the spinal cord as well. Neuroinflammation in the cord could produce a mixture of pain, autonomic dysfunctions, somatovisceral dysfunctions, and/or abnormal motor functions. CRPS is a neurological disorder where many of these dysfunctions can be expressed in a single patient (Schwartzman et al. 2009).

Vascular breakdown or leakage may be a critical step in allowing autoantibodies access to the neuroautoantigens of CRPS patients. Focal accumulations of neuroautoantibodies on target cells are likely to initiate neuroinflammatory responses. Whether the antibody-initiated neuroinflammation remains as discrete foci, or whether the neuroinflammation begins to propagate through the neuraxis, are key questions for understanding the chronicity and spread of CRPS symptoms in a given patient. CRPS shows distinctive patterns of spread throughout the body (van Hilten et al. 2001; van Rijn et al. 2011). Spread of CRPS symptoms to other body sites often occurs in a contiguous fashion. However, it is possible for CRPS symptoms to appear quickly in non-contiguous locations (van Hilten et al. 2001). Both types of CRPS spreading behavior could be linked to the establishment and spread of neuroinflammation within the neuraxis. At this point in time, how CRPS symptoms spread within the neuraxis is primarily known from an analysis at the symptom level. Cellular and molecular knowledge about the spread of neuroinflammation within the neuraxis in other disorders comes from the neuroimaging of human patients, as well as from animal model studies of neuropathic pain.

Spreading neuroinflammation

Numerous studies have revealed that neuroinflammation does not always remain confined to specific foci in the CNS.
Cascades of intercellular signaling allow neuroinflammation to migrate either anterograde or retrograde from primary sites of neuroinflammation.

Banati et al. (2001) utilized radiolabeled PK11195 as a biomarker to image spreading neuroinflammation in the CNS in response to peripheral nerve injury. They determined that peripheral nerve injury frequently leads to microglial activation at the first order synapse of injured neurons. In a minority of cases, neuroinflammation migrated transsynaptically to the second order synapse, located in higher levels of the CNS, such as the thalamus. Banati (2002) also postulated that by altering neurotransmission and the coding of neuroinformation, neuroinflammation at the first and second order synapses could become drivers for higherorder functional changes within the nervous system.

In concise terms, Banati's principles are:

a. Neuroinflammation can spread to second order synapses via remote neuroimmune activation.

b. Neuroinflammation may be the physiological driver for functional pathologies at third order synapses, as well as higher-order integrative processes (Banati 2003).

Hereafter, we refer to paths of spreading neuroinflammation in the CNS as neuroinflammatory tracks (Fig. 2). This term refers to discrete pathways of neuroinflammation that involve anatomical linkage between primary and secondary foci of neuroinflammation.

The ontogeny of posttraumatic neuroinflammatory tracks was first documented in rodents by Banati et al. (1997). Later, Banati et al. (2001) determined that a cohort of chronic pain patients, who had suffered an antecedent peripheral nerve injury, exhibited persistent microglial activation in their contralateral thalamus. Banati et al. (2001) proposed that neuroinflammation had migrated from first order synapses in the spinal cord in these patients, to second order nociceptive synapses, which are located within the contralateral thalamus. Banati (2003) have also obtained evidence for remote neuroimmune activation (also referred to as transsynaptic microglial activation) in a number of neurological disorders.

Saab and Hains (2009) have discussed cellular and molecular mechanisms for such remote neuroimmune activation. Release of the cytokine CCL21 from the terminal of first order nociceptive neurons is capable of initiating secondary sites of neuroinflammation where the nociceptive neurons terminate, such as the VPL nucleus of the thalamus. Saab and Hains (2009) have postulated the neuroinflammation in the thalamus drives dysrhythmias in thalamocortical loops, which can be detected using EEG (Saab, 2012), thus producing a central generator for neuropathic pain.

Neuroautoantibodies in CRPS patients could modify, exaggerate, and sustain this type of remote neuroimmune 


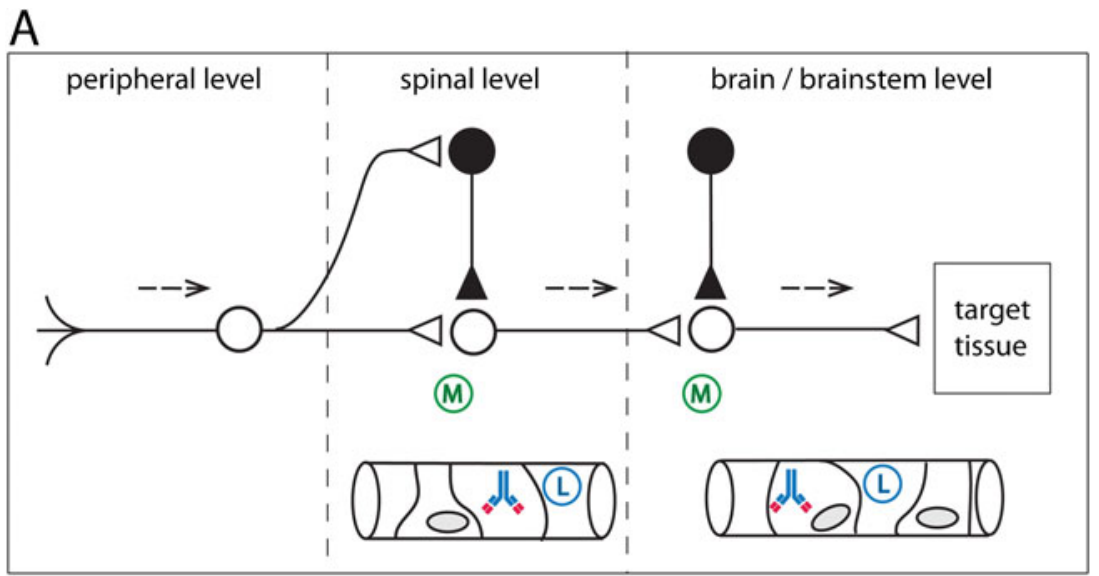

B
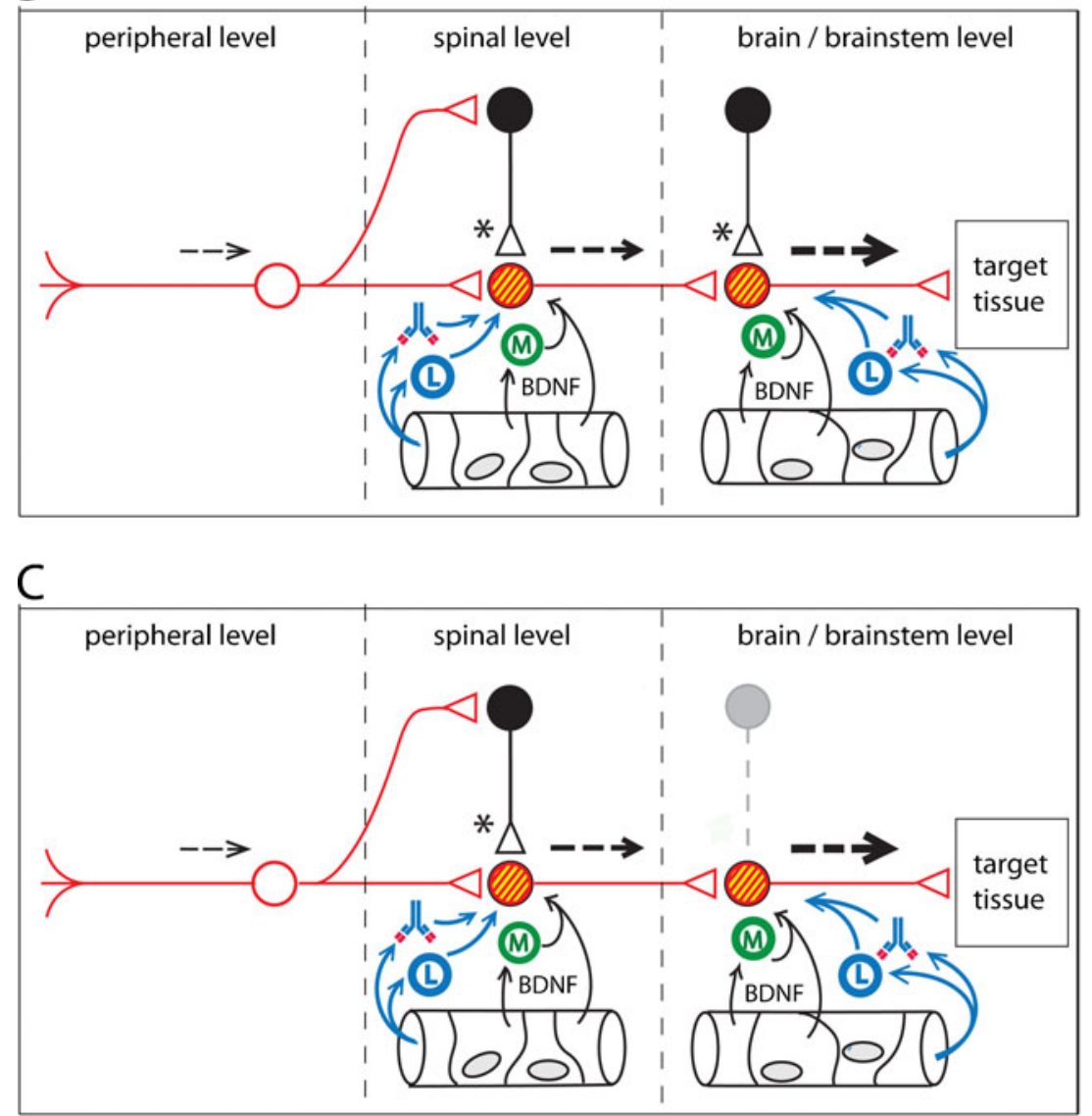

activation (Fig. 1). In this regard, remote neuroimmune activation via neuronal projections may account for the spread of microglial and astroglial activation within the spinal cord of CRPS patients (Del Valle et al. 2009; van Rijn et al. 2011). It has also been hypothesized that spinal neuroinflammation could spread to supraspinal sites in CRPS patients via remote neuroimmune activation to initiate movement disorders (Cooper and Przebinda 2011; Cooper 2011b).
Neuroinflammation and central sensitization

The term "central sensitization" is often used to describe changes in the central nervous system associated with the establishment and progression of the neuroinflammatory diseases. In an experimental model of peripheral inflammatory pain (Roberts et al. 2009), neuroinflammation spreads from the first order synapse, located in the spinal cord, to a second-order synapse in the rostroventromedial medulla 
Fig. 2 A conceptual model for functional pathophysiologies arising from neuroinflammatory tracks. Neuroinflammation leads to a loss of sensory gating, as well as excessive gain, in sensorimotor pathways. a. Normal sensory processing (dotted lines). GABAergic interneurons provide inhibitory tone at both first order and second order synapses. Non-activated microglia (M) lie in the CNS parenchyma. Leukocytes (L) and autoantibodies are present in the CNS vasculature. b. Peripheral injury initiates anterograde central sensitization. Microglial activation takes place in response to cytokines released at the terminals of injured primary afferents (first order synapse). Breakdown of the blood-brain barrier (or the blood-spinal cord barrier) allows activated leukocytes and autoantibodies to extravasate into the parenchyma of the CNS, leading to autoimmune mediated neuroinflammation. Release of Brain-Derived Neurotrophic Factor (BDNF) from plasma, endothelial cells, and activated microglia alter chloride homeostasis in postsynaptic neurons. Accumulation of intracellular chloride (yellow) results in synaptic conversion, changing GABAergic and glycinergic synapses from inhibitory tone to excitatory tone (Huberfeld et al. 2007; Price et al. 2009; Cooper and Przebinda 2011). Sensory gating is lost at the first order synapse. Graded sensory transmission is lost within the neural circuit. Upregulation (red) of excitatory transducers (e.g. glutamate receptors) results an increase in excitatory tone. Functional changes in the circuit result in a collapse of feedforward inhibition and filtering at the first-order synapse (pain gate in the nociceptive circuit) (Cooper and Przebinda 2011). Neuroimmune activation spreads to the second order synapse through the release of the cytokine CCL21 from the terminals of first order neurons (Saab and Hains 2009). Small sensory stimuli provoke large (thick dotted arrows) volleys in second order neurons. c. Central injury to the CNS can result in retrograde central sensitization via neuroimmune activation. Loss of inhibitory interneurons (grey outlines), either by autoimmune attack (e.g. Stiff-Person Syndrome) (Sandbrink et al. 2003; Rokocevic and Floeter 2012), or by metabolic crisis (e.g. hypoglycemia, hypoxia, or excitotoxicity), permanently reduces inhibitory tone in the sensorimotor circuit. These conditions, and/or the conditions illustrated in panel B, may contribute to post-traumatic dystonias, non-dermatomal pain distributions, autonomic, and/or somatovisceral disorders, in patients with autoimmune-mediated neuroinflammation, even after the initial neuroinflammatory event subsides

(RVM). Sensitization of first order and second order trigeminothalamic neurons, for instance, may contribute to the ontogeny of migraine (Borsook et al. 2006; Noseda et al.2011). Non-dermatomal patterns of pain can be generated by neuroinflammation within the thalamus (Burstein et al. 2010).

In CRPS patients, features of central sensitization appear to involve a complex set of neuroinflammatory responses involving NMDA receptors, glial cell activation in the spinal cord (Kiefer et al. 2008; Del Valle et al. 2009), as well as the release of pro-inflammatory cytokines from neurons, glia (Munts et al. 2008), and leukocytes (Alexander et al. 2012).

Extravasation of leukocytes and exudation of autoantibodies into the parenchyma of the nervous system are key features of central sensitization during a neuroautoimmune attack of the CNS. The autoimmune attack is focused on sites where autoantibodies bind. Autoimmune attack can potentially lead to the loss of GABAergic inhibitory interneurons (e.g. Stiff-person Syndrome) (Rokocevic and Floeter 2012). In addition, BDNF released from endothelial cells
(Bayas et al. 2002) or activated microglia in a neuroinflammatory site (Trang et al. 2012) can produce a downregulation of the potassium-chloride co-transporter, KCC2 (Blaesse et al. 2009; Trang et al. 2012), which plays a critical role is maintaining transmembrane chloride gradients in CNS neurons.

Reduction of the transmembrane chloride gradient in the post-synaptic neuron leads to conversion of inhibitory tone to excitatory tone (asterisks in Fig. 2). KCC2 downregulation appears to help BDNF promote neuronal survival (Huberfeld et al. 2007). By converting GABAergic and glycinergic synapses to excitatory function, BDNF promotes increased $\mathrm{Ca}^{2+}$ influx into the affected neurons. Although these dynamics may help neuronal survival (Huberfeld et al. 2007), the resulting synaptic conversion can potentially provoke major disruptions in the logic of neural circuits, leading to major dysfunctions in sensory, motor, and autonomic processes (Cooper and Przebinda 2011) (Fig. 2).

Major nerve injuries in rodents and humans are known to result in leukocyte infiltration into the PNS and CNS (Stoll and Bendszus 2010). Exudation of autoantibodies into the parenchyma of basal ganglia is hypothesized to initiate neuroinflammation and movement disorders (Ahlskog et al. 2001; Citak et al. 2004). Peripheral nerves injury can result in a transient breakdown of the blood-spinal cord barrier and the blood-brain barrier (Beggs et al. 2010). The transient breakdowns in these barriers can be blocked by local anesthesia, indicating that the transient increase in capillary permeability in the CNS is dependent on peripheral nerve activity.

Do minor peripheral nerve injuries in CRPS patients trigger the extravasation of leukocytes and the exudation of autoantibodies from blood vessels, leading to the initiation of persistent sites of neuroinflammation in the PNS, autonomic nervous system, and CNS? Later in this paper, we discuss whether modern neuroimaging methods will be able to answer this question.

\section{CRPS movement disorders}

CRPS researchers have utilized symptomatology to create validated diagnostic criteria for CRPS (Harden et al. 2010). Using a set of four major symptom categories (amplified pain, autonomic dysfunction, motor dysfunction, sudomotor/trophic changes), a set of diagnostic criteria has been identified, which is both sensitive and specific to identify CRPS (Harden 2010). The movement disorders associated with CRPS include fixed dystonia, myoclonus, and tremors. Approximately $80 \%$ of CRPS patients have paresis in their affected body limbs (Birklein et al. 2000).

The nosological effort to define the movement disorders of CRPS as being either physiologic (organic) or psychogenic (non-organic) has been long and controversial (Reedijk et al. 
2008; Munts and Koehler 2010). Recently, a group of movement disorder specialists, who have studied fixed dystonia in CRPS patients, have stated:

"Traditional medical dualism polarizes opinion as to whether fixed dystonia is best characterized as a psychogenic or an organic disorder. ... On the basis of our cases and previous data with regard to amputation in complex regional pain syndrome type I (CRPSI), we speculate that fixed dystonia is itself part of the spectrum of body integrity identity disorders."

Toward the end of their paper, Edwards et al. (2011) state:

"The theory outlined above generates some testable hypotheses regarding the pathophysiology of fixed dystonia that attempt to move beyond the dualistic battle between classifying these patients as organic or psychogenic toward a more integrated view of brain dysfunction in this enigmatic disorder."

This statement represents a major conceptual shift in the differential diagnosis of fixed dystonia. The statement suggests that the fixed dystonias should be reanalyzed in terms of observable neuropathologies. Indeed, distortion of the body schema has been detected in CRPS patients using fMRI (Maihöfner et al. 2003, 2004, 2007). Deficient drive from the parietal cortex to the supplementary cortex and motor cortex is also detected (Maihöfner et al. 2007), leading to "neglect-like" motor symptoms (Galer et al. 1995; Kolb et al. 2012).

Psychogenic was originally coined to indicate nonorganic phenomena "originating in the mind" (Lewis 1972). Using the original semantic of the term "psychogenic", it is difficult to interpret distortions of somatosensory maps in CRPS patients as evidence for psychogenic disease. Evidence for body schema distortions, for instance, has been detected in patients with low back pain (Bray and Moseley 2011). Widespread allodynia can be produced in rodent models by inducing extensive neuroinflammation within the thalamus (Burstein et al. 2010). Thus, non-dermatomal pain distributions, or distortions of the body schema in the somatosensory cortex, cannot be used as positive diagnostic evidence for psychogenic illness. Body schema distortion or thalamic neuroinflammation provide physiological alternatives to the differential diagnosis.

The need of obtaining an accurate differential diagnosis for a disorder is different from the goal (and/or timescale) of obtaining a mechanistic explanation for a disorder. As a consequence, the clinical goals for obtaining a differential diagnosis of a disease state, versus obtaining a mechanistic explanation for the disease state, should not be conflated. In the absence of positive diagnostic evidence for a presumed etiology, the term "idiopathic" should be employed for the symptom, rather than "medically unexplained symptom." This later term biases differential diagnosis towards the unwarranted conclusion of psychogenic illness. From a nosological perspective, it is unclear whether movement disorders that are linked to neuroinflammation should be classified as organic movement disorders, functional movements disorders, or both.

\section{Re-conceptualizing CRPS symptoms}

Can the symptoms of CRPS be re-classified in terms of mechanism/processes, rather than presumed etiology? Without a complete knowledge of how symptoms develop with time, perhaps it is most effective to elucidate whether specific physiological states are present in a given patient. In terms of evaluating neuroautoimmune mechanisms for CRPS symptoms, it is useful to consider five interconnected processes: (a) autoimmune seroconversion, (b) amplification of autoimmune cells and autoantibodies, (c) autoimmune attack, (d) neuroimmune activation, and (e) functional and structural disruption of neural networks.

CRPS symptoms might be categorized by using one or more of the following mechanistic processes and concepts, which could result in the type of lesions indicated:

- Infiltration of autoantibodies into nervous tissues (functional lesion)

- Infiltration of leukocytes into nervous tissues (structural/ functional lesion)

- Focal sites of cytokine imbalance (functional/dynamic lesion)

- Remote neuroimmune activation of glia (structural/ functional lesion)

- Breakdown of blood brain barrier (structural lesion)

- Loss of inhibitory tone (functional lesion)

- Excessive loop gain in neural circuits (dynamic lesion)

- Thalamic neuroinflammation (structural/functional lesion)

- Loss of sensory gating (functional lesion)

- Synaptic conversion (functional lesion)

- Thalamocortical dysrhythmias (dynamic lesion)

- Distortion of a somatotopic map (structural/functional lesion)

- Altered connectivity within the brain (dynamic/functional lesion)

By utilizing the concepts of neuroinflammatory lesions, dynamic lesions, and functional lesions, the differential diagnosis focus shifts away from establishing a psychogenic versus physiologic etiology for a given symptom, toward a more mechanistic analysis of the multiple functional and structural pathologies that are common in CRPS patients. 
Linking neuroautoimmunity with sensorimotor dysfunction

Neuroinflammation provides a wide range of mechanisms to alter sensorimotor function. A recent publication describes a form of chorea in three individuals with nonketotic hyperglycemia (Wang et al. 2009). The authors hypothesize that circulating autoantibodies enter the parenchyma of the basal ganglia as a consequence of hyperglycemia shock to the microvasculature, resulting in a breakdown of the local brain-brain barrier. Microvascular lesions in the basal ganglia were detected with MRI and gadolinium contrast. In one patient the lesion expanded from the right globus pallidus to include the right putamen (Wang et al. 2009):

"These results, together with distinctive distribution of the lesions affecting the putamen, globus pallidus and the head of the caudate without involving the anterior limb of internal capsule, suggest that the autoimmunemediated inflammatory process against basal ganglia neurons may take part in the pathogenesis of some patients with NKH (nonketotic hypoglycemia) induced HC-HB (hemichorea-hemiballism). Conceivably, the basal ganglia might be susceptible to autoimmune attack by anti-GAD65 or other autoantibodies via opening of the blood-brain barrier by hyperglycemia-related hyperosmolality."

The movement disorders in these patients might also be caused by immune complexes activating microglia in the basal ganglia to produce pro-inflammatory cytokines, which in turn induce neuronal dysfunction.

The authors hypothesize that neuroinflammation in these patients results from vascular lesions. Localized breakdown of the blood-brain barrier allows autoantibodies to infiltrate into the parenchyma of the basal ganglia. In this context, the neurovascular lesion is both an organic lesion, as well as a functional lesion. Structural features of the neurovascular lesion are often visible by MRI. Functional changes produced by the neurovascular lesion are evident in the development of the movement disorder. In this case, the neurophysiological mechanisms by which autoimmune-mediated neuroinflammation in the basal ganglia creates the functional pathology (i.e. hemichorea) remain unknown. Microvascular lesions in the brain and spinal cord may be a common way for individuals with neuroautoimmune disorders to have transient neurological and neuropsychiatric events, including movement disorders (Mayer et al. 2010).

The above case illustrates the inherent difficulty of using "medically unexplained symptoms" as a positive diagnostic term. In the previous example, positive radiological evidence provides a plausible etiology of the movement disorder, as well as a positive explanation for the positive response to pharmacological intervention. However, what would the clinical diagnosis be if MRI were not available, and an actual neurovascular lesion were not visible with Xray or $\mathrm{CT}$ ? In the absence of evidence for such structural changes, a practitioner applying the DSM-IV diagnostic criteria could easily misdiagnose the patient as having a motor conversion disorder. Thus, the clinical diagnosis, as well as the subsequent treatment scheme, hinges on the inherent instrumental sensitivity (sometimes marginal) of the neuroimaging modality used to detect neurovascular disruption.

Autoimmunity and movement disorders

Neurologists and psychiatrists have commented that certain individuals appear to be pre-disposed to certain disorders. Some of these individuals may have autoimmune disorders (Appenzeller et al. 2011; Vincent et al. 2011; Irani and Vincent 2011). Unusual tardive movement disorders might occur in individuals with autoimmunity. The following study is illustrative.

A cohort of female patients with antiphospholipid antibodies who took oral contraceptive drugs developed chorea (Cervera et al. 1997). The chorea ceased once the medication was discontinued. The authors of this paper concluded that the unique combination of antiphospholipid (aPL) antibodies and oral contraceptives in these patients produced a movement disorder. The authors hypothesized that chorea arose because of changes in blood flow and metabolism in the basal ganglia, produced by the entrance of autoantibodies into the parenchyma of the basal ganglia. This hypothesis of a neuroautoimmune-mediated metabolic disturbance fits well with the functional lesion concept, originally posited by Charcot (1885) to explain the etiological origins of "hysteria."

Instead of autoimmune-mediated neuroinflammation, the term "medically unexplained symptoms" has often been used to describe sensory, autonomic, and motor symptoms of CRPS patients, even though there is an extensive literature describing neurophysiological pathologies and mechanisms in these patients. There are several inherent difficulties with the psychogenic hypothesis. However, absence of evidence is not evidence of absence. In addition, characteristics such as the speed of onset and remission have also been used to assign symptoms to neurological vs. psychogenic causes, with the assumption that neurological disease processes have a longer timecourse than psychogenic ones. In this regard, rapid onset and rapid remission have often been used as criteria for diagnosing psychogenic movement disorders, even though autoimmune disorders are well know for their cyclic character of remission and relapse.

Another salient difficulty in psychogenic hypotheses is that they often conflate a cluster of atypical symptoms with a psychological pathology. This reasoning excludes atypical presentations of physiological processes, including 
neuroinflammation and neuroautoimmunity, from the differential diagnosis. A patient may have an atypical genetic background, leading to atypical symptoms. Alternatively, the person may have a latent immune response to an infectious agent (Söderberg-Nauclér 2012). Or the person may have been placed on a medication that is counter-indicative for a nervous system with focal sites of neuroinflammation, resulting in anomalous motor or sensory phenomena. In addition, prior injuries, surgeries, infectious antigen presentations, or autoantibody production may have 'primed' the neuroimmune system for an exaggerated neuroimmune response (Hains et al. 2010; Frank et al. 2012). Any or all of the above conditions may exist in certain CRPS patients. These alternative etiologies should be considered before assigning a putative psychogenic etiology to any particular symptom. Selflimiting chorea, for instance, has been associated with Parvovirus B19 infection in children (Fong and de Sousa 2006; Grillo and da Silva 2009). In these cases, immune-mediated encephalopathies are thought to underlie the chorea, even though the MRI is unremarkable in these patients (Grillo and da Silva 2009).

In the diagnosis of CRPS, symptoms such as fixed dystonia are often considered to be a "conversion disorder" (Verdugo and Ochoa, 1995, 2000; Hawley and Weiner 2011). Rather than being a conversion disorder of a psychological origin, these fixed dystonic postures may be the functional consequences of a "seroconversion" event, with autoantibodies serving as the initiators of neurological and neuropsychiatric symptoms. Thus, the autoimmune response itself may have been induced by a combination of physical trauma(s) and hormonal changes associated with psychological stressors, perhaps the onset of symptoms themselves, which further enhance the autoimmune response and symptom severity.

Health professionals have often described physical trauma as antecedent events for CRPS, since the condition was first described (Mitchell 1872). However, the role for psychological stressors as an initiator of CRPS, rather than a consequence of the disorder, has not yet been substantiated (de Rooij et al. 2010).

Individuals with CPRS have often been viewed as having a pre-existing susceptibility for exaggerated pain and movement disorders. If true, where does the predisposition originate? One possibility is that this predisposition is related to the presence of autoantibodies to $\beta 2 \mathrm{AR}$ and M2R. Neuroautoantibodies, for instance, have been found to be associated with Sydenham's chorea (Pavone et al. 2006; Brilot et al. 2011), Sjögren's Syndrome (Vincent et al. 2011), as well certain cases of Tourette's Syndrome (Müller et al. 2004; Morer et al. 2008; Dehning et al. 2009). CRPS may have a similar etiology.

Autoantibodies have been shown to be of creating multiple pathologies within the body (e.g. Sjögren's Syndrome). Memory B-cells, and the autoantibodies that they produce, could be the encoded memory of an earlier traumatic event. Autoantibodies can be generated from immune responses to neoplasms (Vincent et al. 2011). The key concept here is that the development of autoimmunity to specific neuroautoantigens may be the initiating event for many cases of CRPS. Psychological stressors, physical trauma, infectious agents, and/or genetic susceptibility could all play a role in the breakdown of self-tolerance, and the onset of an autoimmune response. This set of etiological linkages fits well with documented clinical experience with CRPS (Mitchell 1872; Birklein et al. 2000). Psychological stressors and immunologic priming have been linked to the enhanced activation of microglia to nervous system injury (Frank et al. 2007; Hains et al. 2010).

Imaging neuroinflammation in movement disorder patients

Autoimmune-mediated movement disorders are thought to occur in a number of autoimmune disorders, such as Sjögren's Syndrome (van den Berg et al. 1999; Venegas et al. 2005; Papageorgiou et al. 2007; Min and Youn 2009), Sydenham's chorea (Vincent et al. 2011), and certain cases of Tourette's Syndrome (Morer et al. 2008; Dehning et al. 2009). Methodologies currently exist that may provide clinically useful means to image neuroinflammation in these and other disorders. Using such methods, neuroinflammation might be expected to be found using PET or MR spectroscopy in the basal ganglia of aPL patients, and in these and other neural fields in Sjögren's Syndrome, Sydenham's chorea, and Tourette's Syndrome, among others. It also would be expected that the degree of symptoms, such as tics or chorea, would correlate with the degree of neuroinflammation.

What happens if the predictive biomarkers are "functional changes" in the nervous system? Neuroimaging and neurovisualization methods are now providing such functional biomarkers to identify neurophysiologic disorders. Changes in blood flow in the brain are commonly used to distinguish alterations of connectivity within the brain. In certain cases, regional changes in brain metabolism can be correlated with transient movement disorders (e.g. onset of paroxysmal or itcal dystonias) (Joo et al. 2004; Yoon et al. 2007).

The issue of sensitivity is central to the clinical tractability of using neuroimaging methods to discern the effects of neuroinflammation in movement disorders. In the case of immune-mediated movement disorders, Citak et al. (2004) have commented:

$\ldots$ in our patients with tics, there were no abnormalities on CT or MRI. On the other hand, similar to the patients with Sydenham's chorea, our patients with post-streptococcal tic disorder also showed striatal hypoperfusion in their SPECT images. This might be 
due to the fact that structural imaging studies might not be as sensitive as functional imaging studies for detecting the abnormalities in striatum in Sydenham's chorea and tic disorders.

The difficulty in detecting certain neuroinflammatory lesions in the brain using structural MRI is also illustrated in the following case study (Maeda et al 2010). A diabetic man exhibited a transient hemiballism (lasting 4 days) in his right arm, which was linked to a hypoglycemic crisis. The movement disorder remitted in response to glucose stabilization with insulin, as well as hemodialysis. Death occurred two months after the transient hemiballism, from severe autonomic neuropathy and cardiac arrest. After autopsy, immunohistological analysis revealed activated microglia in the man's left subthalamic nucleus (STN). Within the same STN, no evidence of neurodegeneration or activated astroglia was found. It is not clear whether activated microglia played a causative role in the movement disorder. Microglial activation was present in the STN, while the hemiballism was in remission. This illustrates that activated microglia alone cannot explain the transient hemiballism in this patient. However, the presence of activated microglia in the STN contralateral to the affected limb does suggest that a physiological event did occur in the STN, perhaps leading to a persistent activation of microglial in the affected STN. Notably, the patient's brain MRI remained unremarkable during these transitions (Maeda et al. 2010).

This above case study strongly supports Charcot's prediction (1885) that dynamic and functional lesions in the CNS could serve as drivers for movement disorders. In the above case of hemiballism, the patient apparently suffered from a functional lesion that had a neuroinflammatory component, without appreciable neuronal death within the STN. However, the neuroinflammatory, functional lesion could not be visualized using standard MR imaging techniques (Maeda et al. 2010).

Cell death within the CNS is, of course, a possible outcome of hyperglycemic or hypoglycemic shock. Neuronal cell death may explain why some patients do not experience resolution of their chorea after their metabolism is normalized (Ahlskog et al. 2001, as irreparable damage may have already occurred (Shan et al. 1998). Loss of inhibitory interneurons from autoimmune attack (Rokocevic and Floeter 2012) can contribute to excessive excitability in the excitatory neuron population of deep brain nuclei. This potential outcome to injury and/or neuroinflammation is illustrated is Fig. 2c. Autoimmune-mediated movement disorders are thought to occur in a number of autoimmune disorders, such as Sjögren's Syndrome (van den Berg et al. 1999; Venegas et al. 2005; Min and Youn 2009), Sydenham's chorea (Vincent et al. 2011), and certain cases of Tourette's Syndrome (Morer et al. 2008; Dehning et al. 2009).
Neuroinflammation and movement disorders

Movement disorders occur in a number of disorders that include neuroautoimmune components, such as Sjögren's Syndrome (van den Berg et al. 1999; Venegas et al. 2005; Min and Youn 2009; Alonso-Navarro et al. 2009), Sydenham's chorea (Brilot et al. 2011; Pavone et al. 2006), and certain cases of Tourette's Syndrome (Morer et al. 2008). Autoimmune-mediated neuroinflammation of the basal ganglia is suspected in many of these cases.

Besides autoimmune-mediated movement disorders, peripheral trauma has long been known to be an antecedent of certain movement disorders (Mitchell 1872; Sankhla et al. 1998; van Rooijen et al. 2011). Mitchell (1872) was among the first to recognize a linkage between neuroinflammation and the genesis of movement disorders in CRPS. Although evidence linking neuroinflammation and movement disorders is rapidly accumulating (van de Warrenburg et al. 2007; Simonyan et al. 2010; van Rooijen et al. 2011), it is clear that a complementary set of neuroimaging methods will be needed to visualize the cellular players and metabolism of neuroinflammatory lesions, to determine their putative roles in motor dysfunction. Neuroinflammatory lesions are clearly heterogeneous in their cellular dynamics, their metabolism, as well as their chronicity.

An idiopathic case of hyperekplexia (startle syndrome) is instructive in this regard (van de Warrenburg et al. 2007). A 55-year-old woman initially presented with a stiff neck, as well as retching when she placed a toothbrush into her mouth. These symptoms resolved over a period of two months. The patient subsequently developed bilateral jaw aches when chewing food. This was followed by severe involuntary mouth closure on attempts to eat. Two months later, she noticed that her arms and shoulders would jerk in response to unexpected sounds or on touching her face. One month later, her left arm became stiff and tended to assume abnormal postures. Her gait became more effortful and she tended to veer to the left.

The patient developed upper and lower extremities jerks, as well as trismus. In addition, the patient developed ptosis on the right side. Decreased pinprick sensation around the nose, upper and lower lip, and forehead; tight and overactive masseter muscles with incomplete mouth opening. There was also rigidity and dystonic posturing of the left arm with mild distal weakness; jerks that were elicited by taps on nose, jaw, forehead, crown and neck and that consisted of bilateral shoulder abduction and forearm flexion with additional posturing of the face. Following symptomatic treatment with medications, most brainstem abnormalities gradually ceased. However, the hyperekplexia persisted for 10 years. The timecourse and characteristics of the patient's symptoms were interpreted as a possible case of selflimiting brainstem neuroinflammation (van de Warrenburg 
et al. 2007). The authors also suspected that the neuroinflammation was autoimmune-mediated.

The movement disorder symptoms of the above patient have important similarities to certain cases reported by Sims et al. (2012) (see Movies S3 (fixed dystonia that remits) and S4 (paroxysmal dystonic attacks) in this paper). These cases, as well as the specific pattern of progression of symptoms in the above case of idiopathic symptomatic hyperekplexia, may involve migration of neuroinflammation from sites of peripheral nerve injury into the brainstem. The specific symptoms suggest that the trigeminal nerve may be involved. Several papers have documented the onset of cranial dystonias following dental procedures or other trauma to tissues in the oral cavity (Sankhla et al. 1998; Schrag et al. 1999). Migration of neuroinflammation from an injured branch of the trigeminal nerve into first-order and secondorder synapses located in the brainstem might serve as drivers for the genesis of such movement disorders (Sims et al. 2012).

Brainstem neuroinflammation was previously considered to explain a case of hyperekplexia, apparently starting from an insect bite on the lower right leg (Kellett et al. 1998). In this patient, movement disorders involving the right leg occurred before generalized body spasms. Migration of neuroinflammation from the spinal level to the reticular formation might provide a mechanism to explain the chronicity and progression of these symptoms. Kellett et al. 1998 postulated a pathology involving glycinergic synapses in the brainstem. A collapse of transmembrane chloride gradients in post-synaptic neurons (Cooper and Przebinda 2011), resulting from neuroinflammation in the brainstem, might account for a loss of glycinergic tone and GABAergic inhibitory tone (Fig. 2c), possibility producing symptomatic hyperekplexia.

\section{Ascending and descending neuroinflammation}

As discussed earlier, the formation of neuroinflammatory tracks, as well as a concomitant the loss of inhibitory synapses and/or interneurons, may allow certain neural pathways to become sensitized centrally. Post-traumatic dystonias and CRPS dystonias might evolve in this manner (Cooper 2011a). Neuroinflammation spreading to second-order synapses in supraspinal centers provides a potent mechanism to destabilize feedback circuits, such as those involved in proprioception, nociception, and autonomic functions. Prolonged sensitivity may result from the loss of inhibitory tone in the affected neural pathway (Rossi et al. 2012).

Remote neuroimmune activation could establish secondary foci of neuroinflammation in the motor cortex (Cooper 2011a), reticular formation, red nucleus, thalamus, cerebellum, rostral ventrolateral medulla (RVM), periaqueductal grey (PAG). Once inhibitory tone is diminished in two or more locations, decompensation is likely to occur, leading to excessive gain in both feedforward and feedback neural circuits (Prescott et al. 2006; Cooper and Przebinda 2011).

In addition to excessive gain in nociceptive, autonomic, and motor circuits, timing errors could also occur in these pathways. Loss of inhibitory tone at the cellular level could easily lead to abnormal time-to-fire behaviors in proprioceptive neurons. This could lead to errors in limb perception, as well as the regulation of postural tone. Body schema distortions might also be generated by neuronal coding problems. We suggest here that neuroinflammatory tracks may be especially potent in producing information processing errors. In feedback systems, two interacting sites within the neuraxis, each with neuroinflammation, may be unable to compensate for corrupted information produced in the other site. Neuroinflammation driven abnormalities in neuronal firing and timing could potentially result in a host of symptoms, which are now being termed functional sensory and motor disorders, instead of psychogenic disorders (Edwards et al. 2012). Such functional sensorimotor integration dysfunctions are prevalent, and sometimes extreme, in CRPS patients (Schwenkreis et al. 2005; McCabe and Blake 2007; Lewis et al. 2007; Turton et al. 2007; McCabe et al. 2009; Huge et al. 2011; Cohen et al. 2012). How specific symptoms are viewed and labeled can dramatically influence the course of medical inquiry, patient-practitioner (biosocial) relationships, as well as the patient acceptance of treatment plans (Stone and Edwards 2011; Edwards and Bhatia 2012).

Changes in muscular tone, ranging from paresis to fixed dystonia, may occur in an affected extremity of CRPS patients (Birklein et al. 2000; van Hilten 2010). Such changes have long been viewed as stigmata for "hysteria", psychosomatic, or psychogenic illness. The dystonias of CRPS and other fixed posttraumatic dystonias need to be reanalyzed from the standpoint of neuroinflammation states. Multi-site information coding failures could easily occur in neural circuits affected by multi-focal neuroinflammation. Substantial evidence has been obtained that spinal reflex circuits are disinhibited at the spinal level in CRPS dystonia (Schouten et al. 2003; van de Beek et al. 2002). Loss of chloride-dependent inhibitory tone at the spinal level, resulting from neuroinflammation, could account for some of this disinhibition. We suggest here that an additional loss of inhibitory tone at supraspinal sites, such as the motor cortex (Cooper 2011a; Fig. 3), reticular formation, and/ or red nucleus, might be needed to maximally decompensate spinal motor reflex circuits, and produce fixed dystonia (Cooper MS (2011b).

Whether neuroinflammation spreads from spinal sites to supraspinal sites in CRPS patients is currently unknown. However, if neuroinflammation does spread from the spinal level to supraspinal sites in CRPS patients, disinhibition of excitatory neurons could occur at both levels, leading to tonic activation of motor neurons (e.g. Fig. 3). This could 


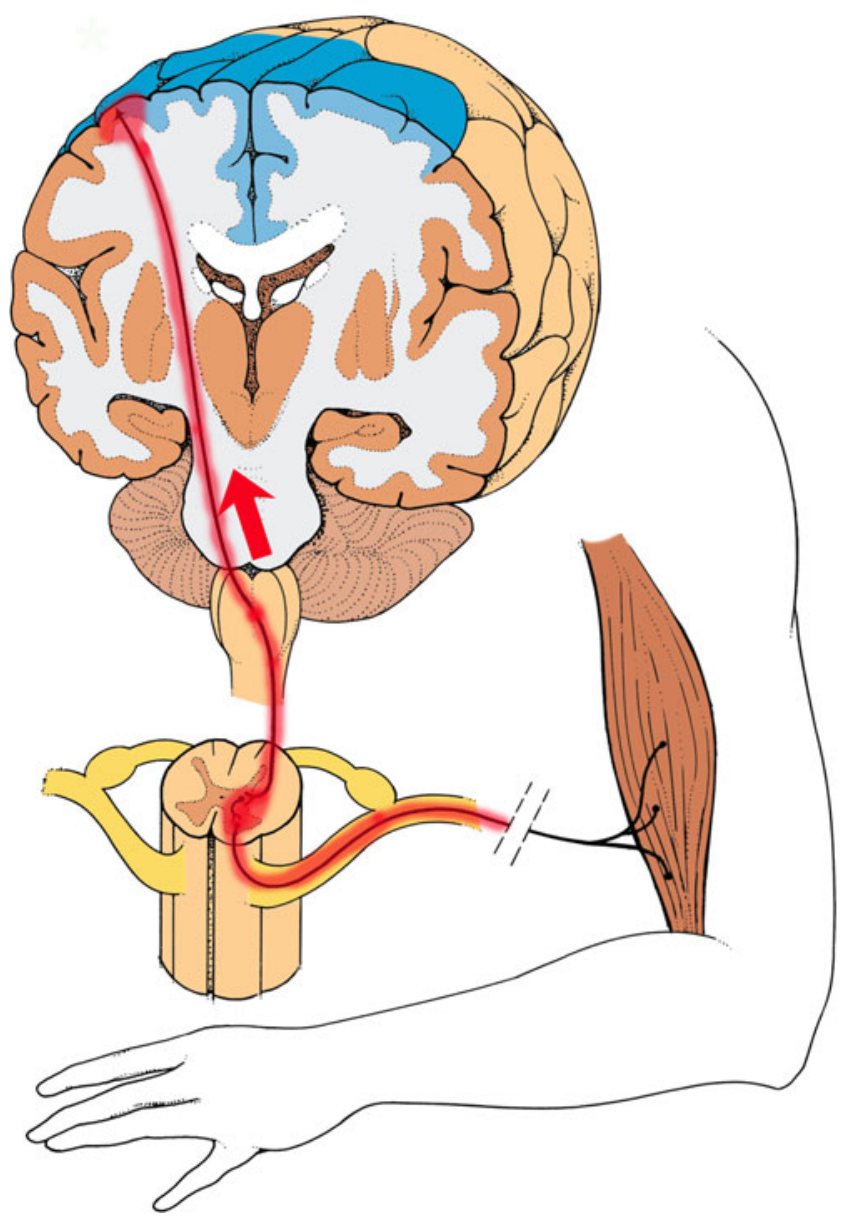

Fig. 3 A neuroinflammatory model for the generation of fixed dystonia. Injury to a peripheral nerve results in inflammation of motor neurons. Neuroinflammation is established in the spinal cord, ipsilateral and segmental to the peripheral nerve injury (Sigel et al. 2007; Lee et al. 2009). Retrograde migration of neuroinflammation (red arrow) occurs via corticospinal neurons, leading to a secondary neuroinflammation site in the motor cortex (Cooper 2011a). Loss of chloridedependent inhibitory tone (similar to panel 2B) and/or an increase in excitatory tone in the cortical and spinal neuroinflammatory foci lead to tonic firing of segmental motor neurons. A neuroinflammationmediated loss of intracortical inhibition in the motor cortex may also be required for the genesis of fixed dystonia. (images modified from The Inner Man ${ }^{\mathrm{TM}}$, Medical Illustrations Company LLC)

potentially produce tremor, myoclonus, or fixed dystonia, depending upon the dynamics and interactions of different motor control centers. Diminished intracortical inhibition has been detected in individuals with posttraumatic fixed dystonias (Espay et al. 2006).

Entrapped nerves are a known etiology of certain cases of cervical and focal dystonias (Ross et al. 1995; Charness et al. 1996; Alafaci et al. 2000; Sun et al. 2009). Neuroinflammatory tracks, with both peripheral and central neuroinflammatory contributions, may play central roles in mediating the ontogeny of these peripherally induced dystonias. To test these hypotheses, it may be soon possible to visualize the distribution and chronicity of both central and peripheral neuroinflammation in movement disorder patients, using a complementary set of emerging neuroimaging methods.

Cytokines and changes within the connectome

Standard neurological examinations are carefully constructed to detect structural defects within the nervous system (Clark et al. 2009). When aberrant reflexive or higher-order neurological behaviors are detected, but cannot be immediately linked to defined structural defects in the nervous system, the aberrant symptoms are often classified as functional pathologies. This distinction, however, is confounded when these pathologies are linked to neuroinflammatory lesions, because neuroinflammation can simultaneously generate both structural and functional changes within the nervous system. Altered cytokine production in peripheral tissues, infiltrating leukocytes, and non-neuronal components of the CNS (glia and endothelial cells) can each become drivers for such changes (Bayas et al. 2002; Ledeboer et al. 2005; Cao and DeLeo 2008; Milligan and Watkins 2009).

It is well established that pro-inflammatory cytokine expression is linked to chronic pain (Milligan and Watkins 2009). Proinflammatory cytokines are also associated with depression, fatigue, sleep disorders, and executive dysfunction (Barbosa et al. 2012; Blume et al. 2011; Capuron and Miller 2011; Mondal et al. 2008). In animal models of neuropathic pain, IL-1 $\beta$ expression becomes elevated in the brainstem, prefrontal cortex, and hippocampus (Apkarian et al. 2006; Besedovsky and del Rey 2011; del Rey et al. 2011). Elevation of IL-1 $\beta$ RNA expression in the hippocampus suggests a direct modulation of a site that is also involved in depression and anxiety (del Rey et al. 2011). In addition, it has been reported that an increase in IL$1 \beta$ expression occurs in the contralateral hippocampus after unilateral sciatic nerve injury (Apkarian et al. 2006).

This later result, along with other earlier reports (Banati et al. 2001; Banati 2002; Roberts et al. 2009), supports the concept that neuroinflammation can become established in supraspinal centers after peripheral nerve injury. Spreading neuroinflammation provides a non-psychogenic etiology to plausibly explain the progression and chronicity of certain disease states, as well as the migration of symptoms to different portions of the body. This mechanistic concept of spreading neuroinflammation within the CNS needs to be incorporated into differential diagnosis of neurological and neuropsychiatric disorders, as well as into the standard use of the Diagnostic and Statistical Manual for Mental Disorders (DSM). This would substantially advance the recognition and diagnosis of neuroinflammatory-mediated functional disorders within the biomedical community.

Cytokine production is a central part of the overlapping and interlocking mechanisms that produce neuroinflammatory disorders, including CRPS. Neuroendocrine alterations and disturbances in the metabolism of monoamines have 
been correlated with cytokine-induced mood and cognitive symptoms (Capuron et al. 2002; Dantzer et al. 2008; Doorduin et al. 2009; Krishnadas and Cavanagh 2012; Kullman et al. 2012). In addition, neuroimaging studies have revealed alterations in specific brain regions during cytokine treatment (Kullmann et al. 2012; Schneider et al. 2012). These changes could contribute to the development of fatigue, depression, and cognitive alterations in patients with neuroinflammation (Raedlera 2011; Schedlowski 2012). In CRPS patients, fatigue, paresis, depression, anxiety, and cognitive impairments are well documented (Mitchell 1872; Birklein et al. 2000; Apkarian et al. 2004).

Numerous structural and functional changes take place in neural networks as a result of altered cytokine expression. To describe effects of neuroimmune activation in neurological and neuropsychiatric disorders, it is important to consider how neuroinflammatory lesions and cytokines affect the connectome, the map of neural connections within the nervous system (Sepulcre et al. 2012). It is also useful to view this connectome as being part of a larger neuromatrix (Iannetti and Mouraux 2010), which is modulated by cellular communication between with a variety of cellular players, including glia, endothelial cells, and leukocytes (Milligan and Watkins 2009; Hughes et al. 2012). Cytokines help mediate these cellular interactions, and thus modify the activities of multi-partiate synapses (i.e. synapses between multiple cell types) within the neuromatrix (De Leo et al. 2006).

At the cellular level, neuroinflammation can produce structural lesions in the following ways: demyelination of axonal fibers (Staff et al. 2010); stripping of synapses from cell bodies by microglia (Perry and O'Connor 2010; Yamada et al. 2011); autoimmune attack on specific interneurons (Rokocevic and Floeter 2012); sprouting of afferent axonal terminals; dieback of sensory nerve endings (Oaklander and Fields 2009); altered dendritic aborizations (Jakubs et al. 2008). These microscopic structural lesions lead to functional changes of local neuronal circuits. Although these structural changes within the connectome may remain largely undetectable using clinical neuroimaging methods, the functional/ behavioral changes that result from these structural changes can be profound. For instance, small fiber neuropathies in the cornea are correlated with the occurrence of painful blepharopasm (Borsook and Rosenthal 2011). In an experimental study, large-fiber neuropathies in peripheral nerves have been correlated with the onset of fixed dystonic postures (Siegel et al. 2007). Clinically, peripheral nerve injury is a known antecedent for many forms of dystonia (Jankovic 2001; 2009), including the fixed dystonias seen in CRPS II patients (50\% of the cohort) (Birklein et al. 2000).

In the CNS, activated microglia can strip neuronal cell bodies of synapses and dendrites, as well as promote demyelination (Perry and O'Connor 2010; Yamada et al. 2011). These microstructural lesions might help to explain the persistent changes in pain processing in children with a history of
CRPS, months after their allodynia has remitted (Lebel et al. 2008). In post-surgical inflammatory neuropathies, reversible demyelination can occur (Staff et al. 2010). Weakness and pain disappear in the affected extremities as remyelination proceeds. The cellular behaviors that underlie demyelination and remyelination should be considered when dealing with the transient expression of neurological and neuropsychiatric symptoms, as well as their long-term remission.

From a number of neuroautoimmune disorders, it is known that the pathological ramifications of neuroautoantibodies entering the parenchyma of the nervous system can be profound. Autoantibodies to $\beta 2 A R$ in CRPS patients, for instance, could bind to $\beta 2 \mathrm{AR}$ receptors located on microglia (Mantyh et al. 1995; Wang et al. 2010), leading to autoimmune attack on these neuroimmune cells, as well as specific types of neurons and astrocytes, which also express $\beta 2 A R$. Aberrant cytokine production from activated microglia is likely to occur if these neuroimmune cells are subjected to an autoimmune attack mediated by autoantibodies and infiltrating leukocytes.

CRPS patient have elevated levels of the pro-inflammatory cytokines IL-1 $\beta$ and IL-6 in their cerebrospinal fluid, as well as reduced levels of the anti-inflammatory cytokines IL-4 and IL-10 (Alexander et al. 2005; Alexander et al. 2005). In peripheral tissues, pro-inflammatory cytokines are found in the affected limbs of CRPS patients (Schinkel et al. 2006), and are enhanced upon transcutaneous electrical stimulation, indicating that neurogenic inflammation is present (Birklein and Schmelz 2008). In general, it is known that antidromic firing of C-fibers (driven by both axonal reflex and dorsal root reflex) can result is CGRP (calcitonin gene related peptide) and SP (substance P) release from the peripheral endings of Cfibers (Willis 1999; Hagains et al. 2010). CGRP and SP sensitize mast cells and the endings of $\mathrm{C}$-fibers. At the peripheral level, local cytokine production can also drive the progression of neuroinflammatory pathologies in many target organs (Li et al. 2009b; Lia et al. 2009). MicroRNA levels are also altered in the blood of CRPS patients (Orlova et al. 2011). These miRNA biomarkers potentially offer a new way for monitoring inflammation in CRPS patients, as well as stratifying patients for therapeutic interventions.

A number of CRPS co-morbidities, including visceral pain, osteopenia, edema, and rashes and ulcerations of the skin, may involve prolonged neurogenic inflammation, as well as its pathological consequences (Oaklander and Fields 2009). Through its corruption of circuit logic within the connectome (Cervero and Laird 1996; Prescott et al. 2006; Cooper and Przebinda 2011), neuroinflammation in spinal, supraspinal, and autonomic centers, might also generate unusual modulations of these diverse pathologies (e.g. episodic or diurnal expression of symptoms). However idiosyncratic the presentation of symptoms may be in individual CRPS patients, it is increasingly difficult to assert a purely 
psychogenic etiology for the co-morbidities of CRPS. The structural and functional consequences of neuroinflammatory lesions provide a logical framework for conceptual modeling and clinical investigation, during the differential diagnosis of unusual neurological or neuropsychiatric symptoms.

\section{Promising neuroimaging approaches}

A MRI-based method that may eventually provide a means to diagnose neuroinflammation is magnetic resonance spectroscopy (MRS). 1H-MRS measures small changes in proton resonance that result from shielding by orbiting electrons. Depending on the chemical structure of the substance being measured, its resonance signature may be detected, and its amplitude (i.e. concentration) can be quantified.

Using this method, a variety of neurochemicals can be identified, including various neurotransmitters and their byproducts (glutamate, glutamine and GABA) membrane components (myo-inositol) constituents of metabolic pathways (choline and creatine) and others with no well understood purpose, but that are sensitive to neuronal function and damage (n- acetyl aspartate, abbreviated as NAA). MRS has been used in a variety of studies to examine chemical changes related to disease processes that may involve neuroinflammation (Holt et al. 2012).

Aside from 1H-MRS, hyperpolarized 13C-MRS provides a means to label carbon atoms in specific metabolically active molecules, which can be injected and are taken up into physiological processes in the body (Rothman et al. 2002). While not radioactive, $13 \mathrm{C}$ can produce an NMR signal that is small, but detectable. The imaging advantage of $13 \mathrm{C}$ is being able to focus on specific metabolic pathways, such as GABA and glutamate, and the ability to quantify their concentrations and changes in concentration as other variables are manipulated. These methods have been used pre-clinically to detect tumors and tumor metabolism. Hyperpolarized 13C-MRS has yet to be utilized to image neuroinflammation in human patients.

As physical properties of the CNS change with neuroinflammation, it might be possible to visualize them using non-invasive imaging methods that depend only on intrinsic properties of the CNS. Using methods such as MRI, the difficulty is often not in the degree of sensitivity, but in specificity. Especially in the case of MRI, the measures that can be obtained are affected by a large variety of factors, and so may differ from the population mean due to a variety of reasons, many of which may reflect properties not involved in the disease process. The specific causes of changes found in MRI data from the CNS can be difficult to infer. Changes in the number or size and type of any CNS cell could contribute to these structural changes, including the size and numbers of either different classes of neurons or glia, as well as their metabolic state (Shan and Pan 2007).
While intriguing, such effects are also often inconclusive as to their mechanisms. Additionally, due to a high degree of individual variability, many imaging protocols require that data be obtained before and after the eliciting event or some intrinsic change in the CNS, which provides a much more sensitive basis to detect effects compared with what is essentially a between-subjects comparison that is found in most physician offices. These two features: (a) lack of specificity and (b) lack of sensitivity for a single imaging sample in an individual patient, remain major barriers to using imaging to identify neuroinflammatory lesions in clinical practice. What is needed is to identify measures that are both sensitive and specific, and can be obtained from a single imaging session.

Recently, magnetic nanoparticles have been used in human patients to detect the infiltration of leukocytes into injured nervous tissues (Stoll and Bendszus 2010; Thorek et al. 2011; Deddens et al. 2012). New contrast agents are being developed for PET/SPECT (Chopra et al. 2012), MRI (Stoll et al. 2012), and MRS imaging (Li et al. 2009a; Witney and Brindle 2010) as well. MRS and diffusion tensor imaging (DTI) can also be used to examine changes in nervous tissues affected by factors such as chronic viral infection (Holt et al. 2012). These rapidly advancing technological developments may also allow neuroinflammation to be imaged in difficult sites, such as the brainstem and spinal cord. Such neuroimaging studies would be hugely beneficial for understanding the establishment and spread of CRPS symptoms.

\section{Conclusion}

In clinical practice, when an organic explanation cannot be found for functional disorders, psychogenic etiologies are often asserted. However, as the understanding of functional disorders improves, it appears that neuroimmune and neuroinflammatory disorders are much more common than previously thought. Neuroautoimmunity combined with neuroinflammation together provide a viable etiology for the relapsing-remitting chronicity, atypical presentation and intensity of neurological and psychiatric symptoms.

When faced with medically unexplained symptoms, health practitioners and patients should actively seek physiological hypotheses, as an alternative to diagnoses of somatoform or conversion disorders. Neuroinflammatory lesions, along with their functional consequences, should always be considered in the differential diagnosis of medically unexplained symptoms, even when commonly used clinical neuroimaging protocols fail to reveal positive evidence of neuroinflammation.

When viewing the symptoms of an individual patient, health practitioners should anticipate that a given patient 
might have a complex, multifocal pattern of neuroinflammation and associated functional symptoms. The presentation of symptoms may be idiosyncratic to that patient, depending on the topographic pattern and severity of neuroinflammation and other variables, often yet undiscovered. However, key mechanistic elements of spreading neuroinflammation could be common to a wide spectrum of patients and disorders. By integrating neuroinflammation and neuroautoimmune concepts into the differential diagnosis of neurological and neuropsychiatric symptoms, a number of functional sensory and motor disorders may become less medically unexplainable than was previously thought. As diagnostic methods continue to improve, it may someday be possible for neuroautoimmuity and neuroinflammation to be commonly assessed as a part of a differential diagnosis of chronic pain syndromes such as CRPS.

Acknowledgments We thank Sam Bloomsburg and Wallis Thompson for their help with manuscript construction. The authors declare that they have no conflict of interest.

Open Access This article is distributed under the terms of the Creative Commons Attribution License which permits any use, distribution, and reproduction in any medium, provided the original author(s) and the source are credited.

\section{References}

Ahlskog JE, Nishino H, Evidente VGH, Tulloch JW, Forbes GS, Caviness JN, Gwinn-Hardy KA (2001) Persistent chorea triggered by hyperglycemic crisis in diabetics. Mov Disorders 16:890-898

Alafaci C, Salpietro FM, Montemagno G, Grasso G, Tomasello F (2000) Spasmodic torticollis due to neurovascular compression of the spinal accessory nerve by the anteroinferior cerebellar artery: Case report. Neurosurgery 47:768-71

Alexander GM, van Rijn MA, van Hilten JJ, Perrault MJ, Schwartzman RJ (2005) Changes in cerebrospinal fluid levels of pro-inflammatory cytokines in CRPS. Pain 116:213-219

Alexander GM, Peterlin BL, Perreault MJ, Grothusen JR, Schwartzman RJ (2012) Changes in plasma cytokines and their soluble receptors in complex regional pain syndrome. J Pain 13:10-20

Alonso-Navarro H, Arroyo M, Parra A, Jiménez-Jiménez FJ (2009) Paroxysmal dystonia associated to primary Sjögren's syndrome. Mov Disord 24:788-90

Apkarian AV, Sosa Y, Krauss BR, Thomas PS, Fredrickson BE, Levy RE, Harden RN, Chialvo R (2004) Chronic pain patients are impaired on an emotional decision-making task. Pain 108:129-136

Apkarian AV, Lavarello S, Randolf A, Berra HH, Chialvo DR, Besedovsky HO, del Rey A (2006) Expression of IL- $1 \beta$ in supraspinal brain regions in rats with neuropathic pain. Neurosci Lett 407:176-181

Appenzeller S, Yeh S, Maruyama M, Barros SM, Carvalho JF (2011) Chorea in primary antiphospholipid syndrome is associated with rheumatic fever. Rheumatol Int. [Epub ahead of print]

Banati RB, Gehrman J, Schubert P, Kreutzberg GW (1997) PK ("peripheral benzodiazepine")-binding sites in the CNS indicate early and discrete brain lesions: microautoradiographic detection of $[3 \mathrm{H}]$ PK11195 binding to activated microglia. J Neurocytol 26:77-82
Banati RB (2003) Neuropathological imaging: in vivo detection of glial activation as a measure of disease and adaptive change in the brain. British Medical Bulletin 65:121-131

Banati RB (2002) Brain plasticity and microglia: is transsynaptic glial activation in the thalamus after limb denervation linked to cortical plasticity and central sensitization? J Physiol Paris 96:289-299

Banati RB, Cagnin A, Brooks DJ, Gunn RN, Myers R, Jones T, Birch $\mathrm{R}$, Anand P (2001) Long-term trans-synaptic glial responses in the human thalamus after peripheral nerve injury. Neuro Report 12:3439-3442

Barbosa IG, Rocha NP, Huguet RB, Ferreira RA, Salgado JV, Carvalho LA, Pariante CM, Teixeira AL (2012) Executive dysfunction in euthymic bipolar disorder patients and its association with plasma biomarkers. J Affective Disorders 137:151-155

Bayas A, Hummel V, Kallmann BA, Karch C, Toyka KV, Rieckmann P (2002) Human cerebral endothelial cells are a potential source for bioactive BDNF. Cytokine 19:55-58

Beggs S, Lui XJ, Kwan C, Salter MW (2010) Peripheral nerve injury and TRPV1-expressing primary afferent $\mathrm{C}$-fibers cause opening of the blood-brain barrier. Molecular Pain 6:74

Besedovsky HO, del Rey A (2011) Central and peripheral cytokines mediate immune-brain connectivity. Neurochem Res 36:1-6

Birklein F, Schmelz M (2008) Neuropeptides, neurogenic inflammation and complex regional pain syndrome (CRPS). Neurosci Lett 437:199-202

Birklein F, Riedl B, Sieweke N, Weber M, Neundörfer B (2000) Neurological findings in complex regional pain syndromes analysis of 145 cases. Acta Neurol Scand 101:262-269

Blaes F, Tschernatsch M, Braeu ME, Matz O, Schmitz K, Nascimento D, Kaps M, Birklein F (2007) Autoimmunity in complex regional pain syndrome. Ann N Y Acad Sci 1107:168-173

Blaesse P, Airaksinen MS, Rivera C, Kaila K (2009) Cation-chloride cotransporters and neuronal function. Neuron 26:820-838

Blume J, Douglas SD, Evans DL (2011) Immune suppression and immune activation in depression. Brain Behav Immun 25:221-229

Borsook D, Rosenthal R (2011) Chronic (neuropathic) corneal pain and blepharospasm: Five case reports. Pain 152:2477-2431

Borsook D, Burstein R, Moulton E, Becerra L (2006) Functional imaging of migraine and the trigeminal system: Applications to migraine pathophysiology. Headache 46(Suppl 1):S32-S38

Bray H, Moseley GL (2011) Disrupted working body schema of the trunk in people with back pain. Br J Sports Med 45:168-173

Brilot F, Merheb V, Ding A, Murphy T, Dale RC (2011) Antibody binding to neuronal surface in Sydenham chorea, but not in PANDAS or tourette syndrome. Neurology 76(17):1508-1513

Burstein R, Jakubowski M, Garcia-Nicas E, Kainz V, Baiwa Z, Hargreaves R, Becerra L, Borsook D (2010) Thalamic sensitization transforms localized pain into widespread allodynia. Ann Neurol 68:81-91

Cao L, DeLeo JA (2008) CNS-infiltrating CD4+ lymphocytes contribute to murine spinal nerve transection-indued neuropathic pain. Eur J Immunol 238:448-58

Capuron L, Gumnick JF, Musselman DL, Lawson DH, Reemsnyder A, Nemeroff CB, Miller AH (2002) Neurobehavioral effects of interferon-alpha in cancer patients: Phenomenology and paroxetine responsiveness of symptom dimensions. Neuropsychopharmacology 26:643-652

Capuron L, Miller AH (2011) Immune system to brain signaling: Neuropsychopharmacological implications. Pharmacol Ther 130:226-238

Cervera R, Asherson RA, Font J, Tikly M, Pallarés L, Chamorro A et al (1997) Chorea in the antiphospholipid syndrome. Clinical, radiologic, and immunologic characteristics of 50 patients from our clinics and the recent literature. Medicine (Baltimore) 76:203-12

Cervero F, Laird JM (1996) Mechanisms of touch-evoked pain (allodynia): A new model. Pain 68:13-23 
Charcot J-M (1885) Lezioni cliniche dell'anno scolastico 1883-84 sulle malatie del sistema nervoso. [Translated by D. Miliotti]. Milan

Charcot JM, Hospice de la Salpê triêre (1892) Clinique des maladies du système nerveux, vol. 1. Progrès Médical, Paris, pp 95-116

Charness ME, Ross MH, Shefner JM (1996) Ulnar neuropathy and dystonic flexion of the fourth and fifth digits: Clinical correlation in musicians. Muscle Nerve 19:431-437

Chopra A, Shan L, Eckelman WC, Leung K, Latterner M, Bryant SH, Menkens (2012) Molecular imaging and contrast agent database (MICAD): Evolution and progress. Mol Imaging Biol 14:4-13

Citak EC, Gücüyener K, Karabacak NI, Serdaroglu A, Okuyaz C, Aydin K (2004) Functional brain imaging in Sydenham's chorea and streptococcal tic disorders. J Child Neurol 19:387-390

Clark C et al (eds) (2009) Neurology. Wiley-Blackwell, Oxford, England

Cohen HE, Hall J, Harris N, McCabe CS, Blake DR, Jänig W (2012) Enhanced pain and autonomic responses to ambiguous visual stimuli in chronic Complex Regional Pain Syndrome (CRPS) type I. Eur J Pain 16:182-195

Cooper MS (2011a) Nerve injuries and the fixed dystonias of CRPS. Pain Med 12:842-843

Cooper MS (2011b) http://www.youtube.com/watch? $\mathrm{v}=\mathrm{iLmTJpYkAKo \& context=C383e6fdADOEgsToPDskJRii}$ rrtaHGrOjN4CBsn4L

Cooper MS, Przebinda AS (2011) Synaptic conversion of chloridedependent synapses in spinal nociceptive circuits: Roles in neuropathic pain. Pain Res Treat 2011:738645

Culmsee C (2009) Targeting beta 2-adrenoceptors for neuroprotection after cerebral ischemia: Is inhibition of stimulation best? Anesth Analg 108:3-5

Dantzer R, Capuron L, Irwin MR, Miller AH, Ollat H, Perry VH, Rousey S, Yirmiya R (2008) Identification and treatment of symptoms associated with inflammation in medically ill patients. Psychoneuroendrocrinology 33:18-29

De Leo JA, Tawfik VL, LaCroix-Fralish ML (2006) The tetrapartite synapse: path to CNS sensitization and chronic pain. Pain 122:17-21

del Rey A, Yau H-J, Randolff A, Centeno MV, Wildmann J, Martina M, Besedovsky HO, Apkarian AV (2011) Chronic neuropathic pain-like behavior correlates with IL-1b expression and disrupts cytokine interactions in the hippocampus. Pain 152:2827-2835

de Rooij AM, Perez RS, Huygen FJ, van Eijs F, van Kleef M, Bauer MC, van Hilten JJ, Marinus J (2010) Spontaneous onset of complex regional pain syndrome. Eur J Pain 14:510-513

Deddens LH, Van Tilborg GA, Mulder WJ, De Vries HE, Dijkhuizen RM (2012) Imaging neuroinflammation after stroke: current status of cellular and molecular MRI strategies. Cerebrovasc Dis 33:392-402

Dehning S, Matz J, Riedel M, Kerle IA, Müller N (2009) Symptom exacerbation in tourette syndrome due to bacterial reinfection. J Clin Psychiatry 70:1606

Del Valle L, Schwartzman RJ, Alexander G (2009) Spinal cord histopathological alterations in a patient with longstanding complex regional pain syndrome. Brain Behav Immun 23:85-91

Doorduin J, de Vries EFJ, Willemsen ATM, de Groot JC, Dierckx RA, Klein HC (2009) Neuroinflammation in schizophrenia-related psychosis: a PET study. J Nucl Med 50:1801-1807

Edwards MJ, Alonso-Canovas A, Schrag A, Bloom BR, Thompson PD, Bhatia K (2011) Limb amputations in fixed dystonia: a form of body integrity identity disorder? Mov Disord 26:1410-1414

Edwards MJ, Adams RA, Brown H, Pareés I, Friston KJ (2012) A Bayesian account of 'hysteria'. Brain [Epub ahead of print]

Edwards MJ, Bhatia KP (2012) Functional (psychogenic) movement disorders: merging mind and brain. Lancet Neurol 11:250260
Espay AJ, Morgante F, Purzner J, Gunraj CA, Lang AE, Chen R (2006) Cortical and spinal abnormalities in psychogenic dystonia. Ann Neurol 59:825-34

Fong CY, de Sousa C (2006) Childhood choreaencephalopathy associated with human parvovirus B19 infection. Dev Med Child Neurol 48:526-28

Frank MG, Baratta MV, Sprunger DB, Watkins LR, Maier SF (2007) Microglia serve as a neuroimmune substrate for stress-induced potentiation of CNS pro-inflammatory cytokine responses. Brain Behav Immuno 21:47-59.

Frank MG, Thompson BM, Watkins LR, Maier SF (2012) Glucocorticoids mediate stress-induced priming of microglial pro-inflammatory responses. Brain Behav Immun 26:337345

Galer BS, Butler S, Jensen MP (1995) Case reports and hypothesis: A neglect- like syndrome may be responsible for the motor disturbance in reflex sympathetic dystrophy (complex regional pain syndrome-1). J Pain Symptom Manage 10:385-391

Goebel A (2011) Complex regional pain syndrome in adults. Rheumatology 50:1739-1750

Goebel A, Leite MI, Yang L, Deacon R, Cendan CM, FoxLewis A, Vincent A (2011) The passive transfer of immunoglobulin $G$ serum antibodies from patients with longstanding Complex Regional Pain Syndrome. Eur J Pain 15:504e1-6

Grillo E, da Silva RJM (2009) Childhood chorea-encephalopathy and unremarkable MRI: an association suggesting parvovirus B19 infection. Dev Med Child Neurol 51:759-61

Hagains CE, Trevino LA, He JW, Liu H, Peng YB (2010) Contributions of dorsal root reflex and axonal reflex to formalin-induced inflammation. Brain Res 1359:90-97

Hains LE, Loram LC, Weiseler JL, Frank MG, Bloss EB, Sholar P, Taylor FR, Harrison JA, Martin TJ, Eisenach JC, Maier SF, Watkins LR (2010) Pain intensity and duration can be enhanced by prior challenge: Initial evidence suggestive of a role of microglial priming. J Pain 11:1004-1014

Harden RN (2010) Objectification of the diagnostic criteria for CRPS Pain Medicine 11:1212-1215

Harden RN, Bruehl S, Perez RS, Birklen F, Marinus J, Maihofner C, Lubenow T, Buvanendran A, Mackey S, Graciosa J, Mogilevski M, Ramsden C, Chont M, Vatine JJ (2010) Validation of proposed diagnostic criteria (the "Budapest Criteria") for complex regional pain syndrome. Pain 150:268-274

Harris JC (2005) A clinical lesson at the Salpetriere. Arch Gen Psychiatry 62:470-472

Hawley JS, Weiner WJ (2011) Psychogenic dystonia and peripheral trauma. Neurology 77:496-502

Holt JL, Kraft-Terry SD, Chang L (2012) Neuroimaging studies of the aging of HIV-1-infected brain. J Neurovirol [Epub ahead of print]

Huberfeld G, Wittner L, Clemenceau S, Baulac M, Kaila K, Miles R, Rivera C (2007) Perturbed chloride homeostasis and GABAergic signaling in human temporal lobe epilepsy. J Neurosci 12:9866-9873

Huge V, Lauchart M, Magerl W, Beyer A, Moehnle P, Kaufhold W, Schelling G, Azad SC (2011) Complex interactions of sensory and motor signs and symptoms in chronic CRPS. PLoS One 6:e18775

Hughes JP, Chessell I, Malamut R, Perkins M, Bačkonja M, Baron R, Farrar JT, Field MJ, Gereau RW, Gilron I, McMahon SB, Porreca F, Rappaport BA, Rice F, Richman LK, Segerdahl M, Seminowicz DA, Watkins LR, Waxman SG, Wiech K, Woolf C (2012) Understanding chronic inflammatory and neuropathic pain. NY Acad Sci 1255:30-44 
Iannetti GD, Mouraux A (2010) From the neuromatrix to the pain matrix (and back). Exp Brain Res 205:1-12

Irani SR, Vincent A (2011) Autoimmune encephalitis-new awareness, challenging questions. Discov Med 11:449-58

Jakubs K, Bonde S, Iosif RE, Ekdahl CT, Kokaia Z, Kokaia M, Lindvall O (2008) Inflammation regulates functional integration of neurons born in adult brain. J Neurosci 28:12477-12488

Jankovic J (2001) Can peripheral trauma induce dystonia and other movement disorders? Mov Disord 16:7-12

Jankovic J (2009) Peripherally induced movement disorders. Neurol Clin 27:821-832

Joo EY, Hong SB, Lee EK, Tae WS, Kim JH, Seo DW, Hong SC, Kim S, Kim MH (2004) Regional cerebral hyperperfusion with ictal dystonic posturing: Ictal-interictal SPECT subtraction. Epilepsia 45:686-689

Katzung BG (2001) Basic and Clinical Pharmacology. Lange Medical Books, New York

Kellett MW, Humphrey PR, Tedman BM, Steiger MJ (1998) Hyperekplexia and trismus due to brainstem encephalopathy. J Neurol Neurosurg Psychiatry 65:122-125

Kiefer R-T, Rohr P, Pioppa A, Dieterich H-J, Grothusen J, Koffler S, Altemeyer K-H, Unertl K, Schwartzman RJ (2008) Efficacy of ketamine in anesthetic dosage for the treatment of refractory complex regional pain syndrome: An open-label phase II study. Pain Medicine 9:1173-1201

Kolb L, Lang C, Seifert F, Maihöfner C (2012) Cognitive correlates of "neglect-like syndrome" in patients with complex regional pain syndrome. Pain 153:1063-73

Kohr D, Singh P, Tschernatsch M, Kaps M, Pouokam E, Diener M, Kummer W, Birklein F, Vincent A, Goebel A, Wallukat G, Blaes F (2011) Autoimmunity against the $b(2)$ adrenergic receptor and muscarinic-2 receptor in complex regional pain syndrome. Pain 152:2690-2700

Krishnadas R, Cavanagh J (2012) Depression: an inflammatory illness? J Neurol Neurosurg Psychiatry 83:495-502

Kullmann JS, Grigoleit JS, Lichte P, Kobbe P, Rosenberger C, Banner C, Wolf OT, Engler H, Oberbeck R, Elsenbruch S, Bingel U, Forsting M, Gizewski ER, Schedlowski M (2012) Neural response to emotional stimuli during experimental human endotoxemia. Hum Brain Mapp. doi:10.1002/hbm.22063

Ledeboer A, Gamanos M, Lai W, Martin D, Maier SF, Watkins LR, Quan N (2005) Involvement of spinal cord nuclear factor kappaB activation in rat models of proinflammatory cytokine-mediated pain facilitation. Eur J Neurosci 22:1977-1986

Lebel A, Becerra L, Wallin D, Moulton EA, Morris S, Pendse G, Jasciewicz J, Stein M, Aiello-Lammens M, Grant E, Berde C, Borsook D (2008) fMRI reveals distinct CNS processing during symptomatic and recovered complex regional pain syndrome in children. Brain 131:1854-1879

Lee JW, Siegel SM, Oaklander AL (2009) Effects of distal nerve injuries on dorsal-horn neurons and glia: Relationships between lesion size and mechanical hyperalgesia. Neuroscience 158:904-914

Levey AI, Kitt CA, Simonds WF, Price DL, Brann MR (1991) Identification and localization of muscarinic acetylcholine receptor proteins in brain with subtype-specific antibodies. J Neurosci 11:3216-3226

Lewis A (1972) "Psychogenic": A word and its mutations. Psychol Med 2:209-215

Lewis JS, Kersten P, McCabe CS, McPherson KM, Blake DR (2007) Body perception disturbance: A contribution to pain in complex regional pain syndrome (CRPS). Pain 133:111-119

Li S, Zhang Y, Wang S, Yang J, Ferraris Araneta M, Farris A, Johnson C, Fox S, Innis R, Shen J (2009a) In vivo 13C magnetic resonance spectroscopy of human brain on a cinical $3 \mathrm{~T}$ scanner using [2$13 \mathrm{C}]$ glucose infusion and low-power stochastic decoupling. Magn Reson Med 632:565-573
Li W-W, Guo T-Z, Liang D, Shi X, Wei T, Kingery WS, Clark JD (2009b) The NALP1 inflammasome controls cytokine production and nociception in a rat fracture model of complex regional pain syndrome. Pain 147:277-286

Lia W, Sabsovich I, Guoa T-Z, Zhaoa R, Kingerya WS, Clark JD (2009) The role of enhanced cutaneous IL- $1 \beta$ signaling in a rat tibia fracture model of complex regional pain syndrome. Pain 144:303-313

Maeda K, Katayama Y, Sugimoto T, Somura M, Kajino Y, Ogasawara K, Yasuda H (2010) Activated microglia in the subthalamic nucleus in hyperglycaemic hemiballism: A case report. J Neurol Neruosurg Psychiatry 81:1175-117

Maihöfner C, Baron R, DeCol R, Binder A, Birklein F, Deuschl G, Handwerker HO, Schattschneider J (2007) The motor system shows adaptive changes in complex regional pain syndrome. Brain 130:2671-2687

Maihöfner C, Handwerker HO, Neundörfer B, Birklein F (2004) Cortical reorganization during recovery from complex regional pain syndrome. Neurology 63:693-701

Maihöfner C, Handwerker HO, Neundörfer B, Birklein F (2003) Patterns of cortical reorganization in complex regional pain syndrome. Neurology 61:1707-1715

Mantyh P, Rogers S, Allen C, Catton M, Ghilardi J, Levin L et al (1995) Beta 2-adrenergic receptors are expressed by glia in vivo in the normal and injured central nervous system in the rat, rabbit, and human. J Neurosci 15:152-164

Mayer M, Cerovec M, Rados M, Cikes N (2010) Antiphospholipid syndrome and central nervous system. Clinical Neurology Neurosurg 112:602-608

McCabe CS, Blake DR (2007) Evidence for a mismatch between the brain's movement control system and sensory system as an explanation for some pain-related disorders. Curr Pain Headache Rep 11:104-108

McCabe CS, Cohen H, Hall J, Lewis J, Rodham K, Harris N (2009) Somatosensory conflicts in complex regional pain syndrome type 1 and fibromyalgia syndrome. Curr Rheumatol 11:461-465

Milligan ED, Watkins LR (2009) Pathological and protective roles of glia in chronic pain. Nat Rev Neurosci 10:23-36

Min JH, Youn YC (2009) Bilateral basal ganglia lesions of primary Sjogren syndrome presenting with generalized chorea. Parkinsonism Relat Disord 15:398-399

Mitchell SW (1872) Injuries of Nerves and their Consequences. Lippincott, Philadelphia

Mondal TK, Saha SK, Miller VM, Seegal RF, Lawrence DA (2008) Autoantibody-mediated neuroinflammation: Pathogenesis of neuropsychiatric systemic lupus erythematosus in the NZM88 murine model. Brain, Behavior, and Immunity 22:949-959

Morer A, Lázaro L, Sabater L, Massana J, Castro J, Graus F (2008) Antineuronal antibodies in a group of children with obsessivecompulsive disorder and Tourette syndrome. J Psychiatr Res 42:64-68

Müller N, Riedel M, Blendinger C, Oberle K, Jacobs E, Abele-Horn M (2004) Mycoplasma pneumoniase infection and Tourette's syndrome. Psychiatry Res 129:119-125

Munts AG, Zijlstra FJ, Nibbering PH, Daha MR, Marinus J, Dahan A, van Hilten JJ (2008) Analysis of cerebrospinal fluid inflammatory mediators in chronic complex regional pain syndrome related dystonia. Clin J Pain 24:30-34

Munts AG, Koehler PJ (2010) How psychogenic is dystonia? Views from past to present. Brain 133:1552-1564

Noseda R, Jakubowski M, Kainz V, Borsook D, Burstein R (2011) Cortical projections of functionally identified thalamic trigeminovascular neurons: implications for migraine headache and its associated symptoms. J Neurosci 31:14214-14217

Oaklander AL, Fields HL (2009) Is reflex sympathetic dystrophy/ complex regional pain syndrome type I a small-fiber neuropathy? Ann Neurol 65:629-638 
Ochoa JL, Verdugo RJ (1995) Reflex sympathetic dystrophy. A common clinical avenue for somatoform expression. Neurol Clin 13:351-363

Orlova IA, Alexander GM, Qureshi RA, Sacan A, Graziano A, Barrett JE, Schwartzman RJ, Ajit SK (2011) MicroRNA modulation in complex regional pain syndrome. J Transl Med 9:195

Papageorgiou SG, Kontaxis T, Bonakis A, Kalfakis N (2007) Orofacial dystonia related to Sjogren's syndrome. Clin Rheumatol 26:17791781

Pavone P, Parano E, Rizzo R, Trifiletti RR (2006) Autoimmune neuropsychiatric disorders associated with streptococcal infection: Sydenham chorea, PANDAS, and PANDAS variants. J Child Neurol 21:727-736

Perry VH, O'Connor V (2010) The role of microglia in synaptic stripping and synaptic degeneration: A revised perspective. ASN Neuro 2:e00047

Prescott SA, Sejnowski TJ, De Koninck Y (2006) Reduction of anion reversal potential subverts the inhibitory control of firing rate in spinal lamina I neurons: Towards a biophysical basis for neuropathic pain. Molecular Pain 2:32

Price TJ, Cervero F, Cold MS, Hammond DL, Prescott SA (2009) Chloride regulation in the pain pathway. Brain Res Rev 60:149170

Raedlera TJ (2011) Inflammatory mechanisms in major depressive disorder. Current Opinion Psychiatry 24:519-525

Reedijk WB, van Rijn MA, Roelofs K, Tuijl JP, Marinus J, van Hilten JJ (2008) Psychological features of patients with complex regional pain syndrome type I related dystonia. Mov Disord 23:15511559

Reznikoff G, Manaker S, Rhodes C, Winokur A, Rainbow T (1986) Localization and quantification of beta-adrenergic receptors in human brain. Neurology 36:1067-1073

Roberts J, Ossipov MH, Porreca F (2009) Glial activation in the rostroventromedial medulla promotes descending facilitation to mediate inflammatory hypersensitivity. Eur J Neurosci 30:229241

Rokocevic G, Floeter MK (2012) Autoimmune stiff person syndrome and related myelopathies: Understanding of electrophysiological and immunological processes. Muscle Nerve 45:623-634

Ross MH, Charness ME, Lee D, Logigian EL (1995) Does ulnar neuropathy predispose to focal dystonia? Muscle Nerve 18:606611

Rossi S, Studer V, Motta C, De Chiara V, Barbieri F, Bernardi G, Centonze D (2012) Inflammation inhibits GABA transmission in multiple sclerosis. Mult Scler. [Epub ahead of print]

Rothman DL, Hyder F, Sibson N, Behar KL, Mason GF, Shen J, Petroff OAC, Shulman RG (2002) In vivo magnetic resonance spectroscopy studies of the glutamate and GABA neurotramsmitter cycles and functional neuroenergetics. Neuropsychopharmacology: The Fifth Generation of Progress. American College of Neuropsychopharmacology

Saab CY, Hains BC (2009) Remote neuroimmune signaling: A long-range mechanism of nociceptive network plasticity. Cell 32:110-117

Saab C (2012) Visualizing the complex brain dynamics of chronic pain. J Neuroimmune Pharmacol. doi:10.1007/s11481-012-9378-8

Sandbrink F, Syed NA, Fujii MD, Dalakas MC, Floeter MK (2003) Motor cortex excitability in stiff-person syndrome. Brain 123:2231-2239

Sankhla C, Lai EC, Jankovic J (1998) Peripherally induced oromandibular dystonia. J Neurol Neurosurg Psychiatry 65:722-728

Schedlowski M (2012) Neural response to emotional stimuli during experimental human endotoxemia. Hum Brain Mapp [Epub ahead of print]

Schinkel C, Gaetner A, Zaspel J, Zedler S, Faist E, Schuermann M (2006) Inflammatory mediators are altered in the acute phase of posttraumatic complex regional pain syndrome. Clin J Pain $22: 235-239$
Schneider P, Weber-Fahr W, Schweinfurth N, Ho YJ, Sartorius A, Spanagel R, Pawlak CR (2012) Central metabolite changes and activation of microglia after peripheral interleukin-2 challenge. Brain Behav Immun 26:277-283

Sepulcre J, Sabuncu MR, Johnson KA (2012) Network assemblies in the functional brain. Curr Opin Neurol 25:384-391

Schouten AC, van de Beek WJ, van Hilten JJ, van der Helm FC (2003) Proprioceptive reflexes in patiens with reflex sympathetic dystrophy. Exp Brain Res 151:1-8

Schrag A, Bhatia KP, Quinn NP, Marsden CD (1999) Atypical and typical cranial dystonia following dental procedures. Mov Disord $14: 492-496$

Schwartzman RJ, Erwin KL, Alexander GM (2009) The natural history of complex regional pain syndrome. Clin J Pain 25:273280

Schwenkreis P, Maier C, Tegenthoff M (2005) Motor cortex disinhibition in complex regional pain syndrome (CRPS) - a unilateral or bilateral phenomenon? Pain 115:212-221

Shan D-E, Pan H-C (2007) Presence of activated microglia in a highsignal lesion on T1-weighted MR images: A biopsy sample reexamined. Am J Neuroradiol 28:602

Shan D-E, Ho DMT, Chang C, Pan H-C, Teng MMH (1998) Hemichorea-hemiballism: an explanation for MR signal changes. Am J Neuroradiology 19:863-870

Siegel SM, Lee JW, Oaklander AL (2007) Needlestick distal nerve injury in rats models symptoms of complex regional pain syndrome. Anesth Analg 105:1820-1829

Simonyan K, Ludlow CL, Vortmeyer AO (2010) Brainstem pathology in spasmodic dysphonia. Laryngoscope 120:121-124

Sims AB, Clark VC, Cooper MS (2012) Suppression of movement disorders by jaw realignment. Pain Med 13:731-732

Söderberg-Nauclér C (2012) Autoimmunity induced by human cytomegalovirus in patients with systemic lupus erythematosus. Arthritis Research and Therapy 14:101

Staff NP, Engelstad J, Klein CJ, Amrami KK, Spinner RJ, Dyck PJ, Warner MA, Warner ME, Dyck JB (2010) Post-surgical inflammatory neuropathy. Brain 133:2866-2880

Stoll G, Bendszus M (2010) New approaches to neuroimaging of central nervous system inflammation. Curr Opinion Neurol 23:282-286

Stoll G, Basse-Lüsebrink T, Weise G, Jakob (2012) Visualization of inflammation using (19) F-magnetic resonance imaging and perfluorocarbons. Wiley Interdiscip Rev Nanomed Nanobiotechnol. 2012 Mar 15. [Epub ahead of print]

Stone J, Warlow C, Sharpe M (2010) The symptom of functional weakness: a controlled study of 107 patients. Brain 133:15371551

Stone J, Edwards MJ (2011) How "psychogenic" are psychogenic movement disorders? 26:1787-1788

Stone J, LaFrance WC Jr, Brown R, Spiegel D, Levenson JL, Sharpe M (2011) Conversion disorder: Current problems and potential solution for DSM-5. J Psychosomatic Res 7:369-376

Stone J, Carson A, Adityab H, Prescotta R, Zaubib M, Warlowa C, Shapre M (2009) The role of physical injury in motor and sensory conversion symptoms: A systematic and narrative review. J Psychosomatic Research 66:383-390

Sun K, Lu Y, Hu G, Luo C, Hou L, Chen J, Wu X, Mei Q (2009) Microvascular decompression of the accessory nerve for treatment of spasmodic torticollis: Early results in 12 cases. Acta Neurochir (Wien) 151:1251-1257

Turton AJ, McCabe CS, Harris N, Filipovic SR (2007) Sensorimotor integration in complex regional pain syndrome: a transcranial magnetic stimulation study. Pain 127:270-275

Trang T, Beggs S, Salter MW (2012) Brain-derived neurotrophic factor from microglia: A molecular substrate for neuropathic pain. Neuron Glia Biol 22:1-10 
Thorek DL, Weisshaar CL, Czupryna JC, Winkelstein BA, Tsourkas A (2011) Superparamagnetic iron oxide-enhanced magnetic resonance imaging of neuroinflammation in a rat model of radicular pain. Mol Imaging 10:206-14

van de Beek WJ, Vein A, Hilgevoord AA, van Dijk JG, van Hilten BJ (2002) Neurophysiologic aspects of patients with generalized or multifocal tonic dystonia of reflex sympathetic dystrophy. J Clin Neurophysiol 19:77-83

van de Warrenburg BPC, Cordivari C, Brown P, Bhatia KP (2007) Persisting hyperekplexia after idiopathic, self-limiting brainstem encephalopathy. Mov Disorders 22:1017-1020

Wang J, Li J, Sheng X, Zhao H, Cao XD, Wang YQ, Wu GC (2010) Beta-adrenoceptor mediated surgery-indued production of proinflammatory cytokines in rat microglial cells. J Neuroimmunol 223:77-83

Willis WD Jr (1999) Dorsal root potentials and dorsal root reflexes: A double-edged sword. Exp Brain Res 124:395-421

Witney TH, Brindle KM (2010) Imaging tumour cell metaboism using hyperpolarized 13C magnetic resonance spectroscopy. Biochem Soc Trans 38:1220-1224

van den Berg JS, Horstink MW, van den Hoogen FH, Oyen WJ (1999) Dystonia; a central nervous system presentation of Sjögren's syndrome. Mov Disord 14:374-5

van Hilten JJ (2010) Movement disorders in complex regional pain syndrome. Pain Med 11:1274-1277

van Hilten JJ, van de Beek WJ, Vein AA, van Dijk JG, Middlekoop HA (2001) Clinical aspects of multifocal or generalized tonic dystonia in reflex sympathetic dystrophy. Neurol 56:1762-1765 van Rijn MA, Marinus J, Putter H, Bosselar SR, Moseley GL, van Hilten JJ (2011) Spreading of complex regional pain syndrome: not a random process. J Neural Transm 118:1301-1309

van Rooijen DE, Geraedts EJ, Marinus J, Jankovic J, van Hilten (2011) Peripheral trauma and movement disorders: A systematic review of reported cases. J Neurol Neurosurg Psychiatry 82:892-898

Venegas FP, Sinning M, Miranda M (2005) Primary Sjogren's syndrome presenting as a generalized chorea. Parkinsonism Relat Disord 11:193-194

Verdugo RJ, Ochoa JL (2000) Abnormal movements in complex regional pain syndrome. Muscle Nerve 23:198-205

Vincent A, Bien CG, Irani SR, Waters P (2011) Autoantibodies associated with diseases of the CNS: new developments and future challenges. Lancet Neurol 10:759-772

Wang J-, Wub T, Deng B-, Zhang Y-, Zhang P, Wang Z- (2009) Hemichorea-hemiballismus associated with nonketotic hyperglycemia: A possible role of inflammation. J Neurol Sci 284:198-202, 284

Watkins LR, Hutchinson MR, Milligan ED, Maier SF (2007) "Listening" and "talking" to neurons: Implications of immune activation for pain control and increasing the efficacy of opioids. Brain Res Rev 56:148-69

Yamada J, Nakanishi H, Jinno S (2011) Differential involvement of perineuronal astrocytes and microglia in synaptic stripping after hypoglossal axotomy. Neurosci 182:1-10

Yoon JH, Lee PH, Yoon SN (2007) Subtraction brain SPECT imaging in a patient with paroxysmal exercise-induced dystonia: role of the primary somatosensory cortex. $64: 1652-1656$ 